\title{
Nanoscale Devices for Rectification of High Frequency Radiation from the Infrared through the Visible: A New Approach
}

\author{
N. M. Miskovsky, ${ }^{1,2}$ P. H. Cutler, ${ }^{1,2}$ A. Mayer, ${ }^{3}$ B. L. Weiss, ${ }^{4}$ Brian Willis, ${ }^{5}$ \\ T. E. Sullivan, ${ }^{6}$ and P. B. Lerner ${ }^{2}$ \\ ${ }^{1} 104$ Davey Laboratory, Department of Physics, The Pennsylvania State University, University Park, PA 16802, USA \\ ${ }^{2}$ Scitech Associates LLC, 232 Woodland Drive, State College, PA 16803, USA \\ ${ }^{3}$ Facultés Universitaires Notre-Dame de la Paix, Rue de Bruxelles 61, 5000 Namur, Belgium \\ ${ }^{4}$ Division of Mathematics and Natural Sciences, Pennsylvania State University, Altoona Campus, PA 16601, USA \\ ${ }^{5}$ Chemical, Materials and Biomolecular Engineering Department, University of Connecticut, Storrs, CT 06269, USA \\ ${ }^{6}$ Department of Electrical and Computer Engineering, Temple University, Philadelphia, PA 19122, USA
}

Correspondence should be addressed to N. M. Miskovsky, nmm1@psu.edu

Received 20 December 2011; Accepted 12 February 2012

Academic Editor: Valery Khabashesku

Copyright ( $) 2012$ N. M. Miskovsky et al. This is an open access article distributed under the Creative Commons Attribution License, which permits unrestricted use, distribution, and reproduction in any medium, provided the original work is properly cited.

\begin{abstract}
We present a new and viable method for optical rectification. This approach has been demonstrated both theoretically and experimentally and is the basis fot the development of devices to rectify radiation through the visible. This technique for rectification is based not on conventional material or temperature asymmetry as used in MIM (metal/insulator/metal) or Schottky diodes, but on a purely sharp geometric property of the antenna. This sharp "tip" or edge with a collector anode constitutes a tunnel junction. In these devices the rectenna (consisting of the antenna and the tunnel junction) acts as the absorber of the incident radiation and the rectifier. Using current nanofabrication techniques and the selective atomic layer deposition (ALD) process, junctions of $1 \mathrm{~nm}$ can be fabricated, which allow for rectification of frequencies up to the blue portion of the spectrum. To assess the viability of our approach, we review the development of nanoantenna structures and tunnel junctions capable of operating in the visible region. In addition, we review the detailed process of rectification and present methodologies for analysis of diode data. Finally, we present operational designs for an optical rectenna and its fabrication and discuss outstanding problems and future work.
\end{abstract}

\section{Introduction}

One of the major challenges in the efficient detection and conversion of energy in the electromagnetic spectrum is the development of a broadband device that will collect and rectify electromagnetic radiation from the IR through the visible portion of the spectrum, extending to $10^{15} \mathrm{~Hz}$ and higher. Current MIM and silicon-based Schottky diodes are narrow band devices, rectifying up to the mid-IR range of the electromagnetic spectrum $\left(\leq 10^{14} \mathrm{~Hz}\right)$.

In this paper we present a method for optical rectification, which has been demonstrated both theoretically and experimentally and can be used for the development of a practical rectification device for the electromagnetic spectrum including the visible portion. This technique for optical frequency rectification is based, not on conventional material or temperature asymmetry as used in MIM or Schottky diodes, but on a purely geometric property of the antenna tip or other sharp edges that may be incorporated on patch antennas. This "tip" or edge in conjunction with a collector anode providing connection to the external circuit constitutes a tunnel junction. In these devices the rectenna (consisting of the antenna and the tunnel junction) acts as both the absorber of the incident radiation and the rectifier.

A brief overview of the operation of the optical rectenna is as follows (see Section 2 for more details). Incident radiation is directed to a rectenna, which consists of a receiving antenna (e.g., a patch antenna characterized by its three primary dimensions) with one edge terminated by a tip or sharp-edged structure which is part of a geometrically 
asymmetric metal vacuum/metal tunnel junction (whose surfaces may be coated) with a gap distance $s$. The junction serves as a rectifier of the $\mathrm{AC}$ radiation collected by the antenna, yielding a DC current. The geometric parameters for the antenna are matched to resonance for a narrow band device and, for wide band devices, the geometric parameters are chosen based on energy absorption and energy density. The gap distance is designed so that tunneling time is sufficient for electrons to transit the barrier before field reversal.

Due to the incident radiation $\mathrm{AC}$ currents are induced along the length of the antenna, which produce oscillating charges at the top or edge of the geometrically asymmetric tunneling junction and corresponding image currents in the sample. The presence of the constricted geometry of the tip or edge gives rise to an enhanced field at the tip. These oscillating charges in the tunnel junction induce an AC voltage across the gap. If the induced field is sufficient for field emission, a tunneling current is produced. Due to geometric asymmetry (and appropriate choices of material asymmetry or plasmonic coatings), there is a difference between the potential barriers for forward and reverse bias, which results in a rectified DC current.

This method for optical frequency rectification can be applied to such applications that include but are not limited to photovoltaics (the conversion of photon energy to electrical energy), solar cells which convert solar energy to electrical (US Patent 7,799,988), thermal, or chemical energy, nanophotonics, near-field optics, and IR sensing and imaging including medical and chemical sensors [1, $2]$. Another important application is the transmission and reception of information and energy conversion at optical frequencies. This is significant since the density of transmitted information is greater at higher frequencies; in fact, the density goes as the square of the frequency. For transmission through the atmosphere losses decrease as the frequency increases [2].

However, optical rectification has been a major challenge due to the difficulty in device manufacturing and materials processing at the nanoscale, for example, the fabrication of so-called nantennas on the order of the wavelength of the incident radiation. Furthermore, theoretical understanding of the operation and description of antennas at the nanoscale in the optical regime is only now being studied in a rigorous way taking into account that the behavior of metals in the optical regime, which differs from that at frequencies below the IR. Significant advances in the study of optical antennas has been reported by Bharadwaj et al. [2], Engheta et al. [3], Alda et al., [1] Rutherglen et al. [4], and others.

The uses of rectennas, in the microwave region, have been investigated over the past half century for power transmission and detection [5]. Applications have included long-distance power beaming, signal detection, and wireless control systems. The first receiving device for efficient reception and rectification of microwave power was developed in the early 1960s at Raytheon [5]. Conventional rectennas such as those developed at Raytheon consist of two distinct elements, a dipole antenna plus a separate rectifying diode such as an MIM or Schottky diode [6]. Schottky diodes are generally limited to frequencies $<5 \mathrm{THz}$, and conventional
MIM diodes have been used up to frequencies of about $150 \mathrm{THz}(2 \mu \mathrm{m})$ [6]. The extension of this rectenna into the visible or IR region is a challenge. Aside from the issues regarding the fabrication of well-characterized reproducible nanoscale devices, the response of the rectifying device to optical radiation is a critical part of the operation. This response time consists of several elements: the collective response of the conduction electrons that establish the AC bias; the electrodynamical response of the junction to the changing fields, for example, the RC response time; The "transversal time" for electrons to cross the gap region in the tunnel junction before field reversal. Generally for metals, the collective response corresponds to frequencies well beyond the UV (or periods $\sim 10^{-16} \mathrm{sec}$ ). Below we consider the remaining two in more detail.

In order to estimate the limiting frequency for the tunneling rectifiers used in these nanoscale devices, it is important to introduce the concept of "tunneling time" or "traversal time" applied to barrier transmission through time-dependent barriers. Qualitatively, an electron of a given energy incident on the oscillating barrier "interacts" with the barrier for a time $\tau_{b}$. Consider the two limiting cases. In one limit where the period of the oscillation, $T$, of the radiation is longer than this time of interaction, the electron effectively interacts with a "static" barrier and, hence, can tunnel before the field direction reverses. On the other hand for the limit where the frequency of the radiation is very high with $\tau_{b} \gg T$, then the electron interacts with many cycles of the radiation and the tunneling barrier is essentially unchanged due to the oscillating voltage. In this limit, the tunneling current is comprised of the photoexcited electrons which have absorbed or emitted quanta equal to $n \hbar \Omega$, where $n=1,2, \ldots$ and $\Omega$ is the angular frequency of the incident radiation. The crossover between these two limiting behaviors may be determined by the relationship, $\Omega \tau \approx 1$ [7]. The validity of such a conceptual approach has been the subject of debate and controversy ever since the advent of quantum mechanics and the recognition that there can be particle tunneling through classically forbidden barrier regions. Basically, the problem lies in the difficulty of defining and measuring the traversal time for the conceptually simple time-dependent scattering experiment in which an electron (wave packet) tunnels through a spatially localized barrier and is detected beyond the tunneling region $[8-11]$. Keldysh $[10,11]$ presented another, not altogether incompatible, criterion for adiabatic transmission through the oscillating barrier. The crossover between the multiphoton absorption and the quasi-static tunneling is determined by the parameter $\gamma_{K}=$ $\omega \sqrt{2 m_{e} V_{\text {barrier }}} / e E$, where $m_{e}$ is the electron mass, $e$ the charge on the electron, $E$ is the electric field strength generated by the laser and $\omega$ is the angular frequency of the radiation. In the case of a metal, the height of the barrier is typically identified with the work function and for a semiconductor with the band gap. As would be expected a value of $\gamma_{K} \gg 1$ indicates multiphoton processes dominate (in the over the barrier and/or tunneling process), and $\gamma_{K} \ll 1$ implies single photon processes dominate.

A definitive experiment by Nguyen et al. [12] used a dynamical approach to probe tunneling times in which a 
natural time scale is provided by the laser that is an integral part of the experimental arrangement. The laser incident upon an STM junction (consisting of a W-sharp tip and a polished, flat $\mathrm{Si}$ (111) anode) causes the tunneling and, at the same time, provides a "clock" to measure the duration of the event. Given that the laser-induced electric field is larger near the pointed apex of the tip than at the planar surface of the sample means that the vacuum tunnel barrier will tend to buckle inward (concave) or become thinner for forward bias and balloon outward (convex) or become thicker for reverse bias (see discussion in Section 4.1.3 and Figure 6). Moreover, due to the material asymmetry, there is an additional barrier asymmetry introduced (see Section 4.1.1). Thus, such an STM junction can be a rectifier with a net DC current in the forward bias direction under laser irradiation. It can be argued that, if for a fixed spacing the laser frequency is too high, few electrons will be able to transfer from one electrode to the other during the half of the period when the electric field vector in the laser beam accelerates the tunneling electron. This means that one should observe a cutoff in the strength of the rectified DC signal either (1) when the frequency is increased beyond a critical value while maintaining the tip-to-surface distance s fixed or (2) when the gap width $s$ is increased beyond a characteristic value $s_{e}$, while keeping the laser frequency constant. This latter method was used in these experiments. Specifically, the STM W/Si (111) junction was illuminated by a 1.06-pm yttrium-aluminum-garnet (YAG) laser. In this experiment the tungsten tip was held at a fixed distance $(\sim 1 \mathrm{~nm})$ from the silicon surface, the YAG laser was focused near the junction, and the resulting laser-induced current was measured. We note that while the laser beam irradiated the junction, no external bias was applied to the STM junction, so that the voltage and induced current that were measured in the junction were due solely to the laser field. The tip-to-base gap $s$ was then progressively increased until the laser-induced current vanished. The DC rectified current as a function of gap width for fixed frequency indicated a cutoff distance of about $2.5 \mathrm{~nm}$ for the $1.06 \mathrm{pm}$ YAG laser line.

The experimental results can be described in terms of a simple model that assumes that the particle acts as if it obeys the kinematical equations of motion as the particle traverses the classically inaccessible region defining the barrier at a velocity approximately equal to the Fermi velocity. However, the use of this simple analysis does not presuppose that there is any a priori reason for an electron to act "classically" as it tunnels through a barrier (see Section 4.3 for a more detailed description of the experiment and results).

Using these experimental results we can determine the limiting response time at that frequency. If we assume an average tunneling velocity to be the Fermi velocity $\left(v_{f}\right)$, then $f_{\text {cutoff }}=v_{f} / d$. This analysis predicts that for a $1 \mathrm{~nm}$ gap for a metallic tip and vacuum barrier, the transit time of about $10^{-15}$ seconds, which corresponds to radiation approaching the UV [12]. Using new fabrication techniques (developed by one of the authors, B. Willis) tunnel junction gaps down to $1 \mathrm{~nm}$ can be reproducibly fabricated over $\mathrm{cm}^{2}$ sized areas. This fabrication technique uses selective atomic layer deposition (ALD) to control the tip-collector spacing, a process that is self-limiting at gap separations of $1 \mathrm{~nm}$ for $\mathrm{Cu}$ (see Section 3.2 and [13]). At this spacing, the tunneling time is sufficiently short for electrons to transit the barrier before field reversal (in the visible frequency range), leading to rectification.

However, for the case of a planar MIM/MOM structure, the $\mathrm{RC}$ response time of the junction is limited by parasitic capacitance of the planar MIM structure yielding a practical limit of 10-100 THz [14]. By contrast, point-contact devices (i.e., whisker diodes) have been used in measurements of absolute frequencies up to the green part of the visible spectrum, demonstrating a response time of the order of femtoseconds, orders of magnitude faster than conventional MIM diodes [15]. The asymmetrical, nonplanar geometry of the pointed whisker in conjunction with the flat anode is an essential requirement for increasing the cut-off frequency $\omega_{c}$ of the diode, but inconsistent with the planar geometry of MOM/MIM tunneling theory for which the cut-off frequency is independent of contact area. Indeed the response time $\tau \sim 1 / \omega_{c}=R C$ is independent of contact area for a planar MOM/MIM geometry since $C$, the capacitance of the contact, is proportional to $A$, the contact area, whereas $R$ the resistance is inversely proportional to $A$. On the other hand, for a point contact using a solvable model with a spherical tip, it can be shown that $\omega_{c}$ is proportional to $A^{-1 / 4}$. Thus the sharper the tip, the faster the response time of the diode $[14,16]$. Although mechanical stability of these earlier devices places a limitation on the actual contact area, modern fabrication techniques have overcome the mechanical fragility of previous point contact diodes and issues related to reproducible fabrication of nanoscale devices.

In a series of papers, Mayer et al. [17-21] have used a quantum-mechanical transfer matrix approach for the modeling of a geometrically asymmetric metal-vacuummetal junction that is subject to an oscillating potential. This quantum mechanical scheme accounts for the threedimensional aspects of the problem as well as the time dependence of the barrier. The currents are obtained by solving the time-dependent Schrödinger equation with a Floquet expansion of the wave function. For simulations using a full range of frequencies in the solar spectrum, Mayer et al. investigated how the efficiency of the rectification is affected by the aspect ratio of the tip, the work function of the metallic elements and the occurrence of polarization resonances. His results demonstrate that the rectification of infrared and optical radiation are possible using devices of the type considered in this paper.

In this paper, we present the results of our modeling, characterization, and nanofabrication of a rectenna device acting as both an antenna and rectifier for IR and high frequency optical radiation. In Section 2, we present an overview of the operation of an optical rectenna. Before we present our proposed designs for an optical rectenna, in Section 3 we review the state of development of antenna structures, which can respond to and capture radiation through the visible region. In Section 4, we assess the state of development both experimentally and theoretically of tunnel junctions capable of operating in the visible region. 
Specifically, we review the process of rectification (Sections 4.1.1-4.1.3), present methodologies for analysis of diode data (Section 4.2), review the experimental data confirming rectification in the optical region (Section 4.3), and review the theoretical work on optical rectification (Section 4.4). In Section 5, we present operational designs for an optical rectenna, fabrication, and so forth. Finally, in Section 6, we discuss future work and outstanding problems.

\section{Overview of a New Approach for the Operation and Design of an Optical Rectenna}

We begin by reiterating the elements of the new approach for the operation of the optical rectenna. Incident radiation is directed to a rectenna, which consists of a receiving antenna (e.g., a patch antenna characterized by its three primary dimensions) with one edge terminated by a tip or sharpedged structure which is part of a geometrically asymmetric metal vacuum/metal junction (with a gap distance $s$ ) for the radiation collected by the antenna. The selection of an appropriate structure such as a patch antenna, whisker antenna, or rectenna with a sharp edge coating (such as diamond, BN, GaN, AlN, AlGaN, plasmonic materials, and Cs) is determined by the application and its ability to absorb the incident radiation. The coating can reduce the magnitude of the forward tunneling barrier allowing for enhanced electron emission and rectification. In addition to the nanogeometry employed by whisker antennas, other realizations use patch antennas, which are designed to operate into the IR and higher frequencies and can have extended solid and open geometries (e.g., squares, rectangles, any $n$-sided structure, or others). These rectenna devices can provide a more robust stability. The geometric parameters for the antenna are matched to resonance for a narrow band device and, for wide band devices, the geometric parameters are chosen based on energy absorption and energy density. The gap distance is designed so that the tunneling time is sufficiently short for electrons to transit the barrier before field reversal.

Due to the incident radiation $\mathrm{AC}$ currents are induced along the length of the antenna, which produce oscillating charges at the top or edge of the geometrically asymmetric tunneling junction and corresponding image currents in the sample. The presence of the constricted geometry of the tip or edge gives rise to an enhanced field at the tip. These oscillating charges in the tunnel junction induce an AC voltage across the gap, which may produce a corresponding electric field that is enhanced as compared with the incident field of the electromagnetic wave. If the induced field is sufficient for field emission, a tunneling current is produced. Due to geometric asymmetry (and appropriate choices of material asymmetry or, e.g., plasmonic coatings), there is a difference between the potential barriers for forward and reverse bias, which results in a rectified DC current.

It is important to note that MVM tunnel junction gaps down to $1 \mathrm{~nm}$ can be reproducibly fabricated over $\mathrm{cm}^{2}$ sized areas using selective ALD. In particular, for $\mathrm{Cu}$, the selective ALD process is self-limiting at gap separations of
$1 \mathrm{~nm}$ (see Section 3.2 and [13]). For gap distances of this size, rectifications of radiation with frequencies in the visible range are possible.

\section{State of Development of Optical Antennas (Nanoantennas)}

Optical antennas are devices that collect/transmit optical wavelength free space electromagnetic radiation to/from energy confined to the antenna structure. They are similar in function to their radio, TV, and cellular communication analogs. The common requirement for any antenna structure is that their size be comparable to the wavelength of the collected or transmitted light. For optical antennas this gives a typical dimension of $\sim 100-1000 \mathrm{~nm}$. In addition to the size requirements, to produce an effective output signal, the antenna must be coupled to a device that can respond in a time $t \sim 1 / f$. For optical frequencies through the visible this corresponds to times of $10^{-15}-10^{-13}$ seconds.

The development of optical antennas has been limited by two factors related to the required size and frequency of operation: (1) the ability to fabricate devices with nanometer dimensions and tolerances, and (2) the ability to convert the high frequency signal to a useful output. The continuing development of technologies in the area of materials, nanofabrication tools, and nanolithography techniques has made available the ability to address these technological challenges. This is discussed in detail in the next section and fabricated structures are shown in Appendix A.

The considerable effort directed toward the development of optical antennas has produced a variety of sizes and shapes. Currently these include, but are not limited to, thin wire or whisker, dipole, bow-tie, spiral, and Yagi-Uda (the geometry of this antenna is that of the most common terrestrial TV antenna). The thin wire antenna consists of arrays of vertically aligned CNTs grown by CVD or nanowires produced by template growth on porous anodic aluminum oxide (AAO). The extended shapes, so-called patch antennas, have been fabricated by conventional silicon lithography, e-beam, and ion milling techniques.

\subsection{Thin Wire Antennas Using CNTs and Nanowire Arrays.} It has been demonstrated both theoretically and experimentally that CNTs and nanowires do act as antennae. Burke et al. have shown theoretically that in the infrared and optical regions, where the length of the metallic carbon nanotube (mCNT) or nanowire whisker is wavelengths larger than the incident radiation, the nanowire, and mCNT exhibit the requisite antenna properties [26]. In addition Novotony has discussed the modification of antenna properties when the size (i.e., thickness) of the nanoantenna becomes comparable to the skin depth, an effect which can occur in the IR region of the spectrum $[27,28]$. Kempa et al. have experimentally demonstrated that arrays of vertically aligned multi-walled metallic (mwm) CNTs act as antennae up to the near IR $\geq$ $800 \mathrm{~nm}$ [29]. At frequencies above the IR, the conventional treatments of the electrodynamical behavior and properties of metals are no longer valid. More accurate methodologies 


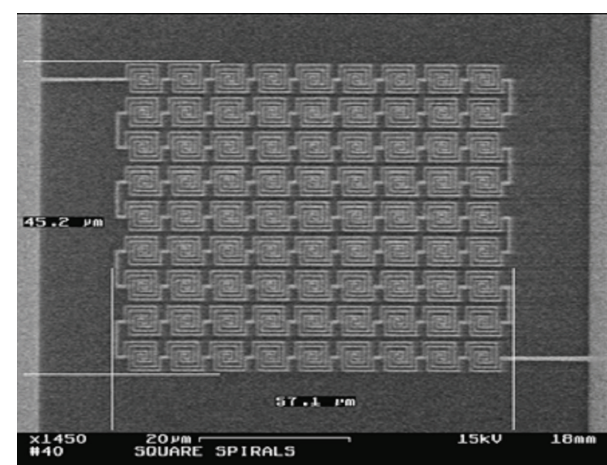

FIGURE 1: SEM micrograph a spiral antenna fabricated by Kotter et al. [22].

taking account the properties of these materials at high frequency at the nanoscale must be used [30-33].

3.2. Patch Antenna Structures. In addition to the nanowire geometry for the whisker antennas, the use of patch antennas can have extended solid and open geometries (e.g., squares, rectangles, bowties, rings, or others) which provide a more robust stability in a practical device. These patch antennas can lead to enhanced antenna absorption properties and signal output [34].

Boreman and his group have fabricated a variety of IR optical patch antenna structures. While their work encompasses electromagnetic radiation from the visible into millimeter regime, of particular note is the group's work in the use of e-beam lithography (EBL) to fabricate dipole antennas coupled to MOM or MIM diodes as detectors operating in the mid-IR $(\sim 10 \mu \mathrm{m})$ [35]. They have fabricated and characterized (for $\lambda=10.6 \mu \mathrm{m}$ radiation) a spiral dipole connected by strip lines [36] and phased array dipoles [37].

The bow-tie antenna consists of two triangles that are oriented tip to tip. The region between the tips enhances the electric field due to the sharp geometry of the structures. This field enhancement was demonstrated by Grober et al. measured in the microwave region [38], Moreover, such antenna arrays of gold have been fabricated on flexible substrates at the Idaho National Laboratory [22]. Moerner and colleagues have extended the work on bow-tie antennas through the IR into the visible [39].

Kotter et al. of the Idaho National Laboratory have fabricated a solar nantenna electromagnetic collector (NEC) [22]. The prototype NEC structures are gold square spiral antennas fabricated on Si wafers using EBL (illustrated in Figure 1). The width of the deposited gold wires is approximately 1000 atoms across. The IR nantennas were designed to operate as a reflective bandpass filter centered at a wavelength of $6.5 \mathrm{um}$. The spectral surface characteristics from 3 to $15 \mu \mathrm{m}$ were studied using spectral radiometer and FTIR analysis methods. The prototype was elevated to a temperature of $200^{\circ} \mathrm{C}$ and its spectral radiance spectrum was compared to blackbody emission at $200^{\circ} \mathrm{C}$. Maximum contrast is over $90 \%$ between emission near $4 \mu \mathrm{m}$ and emission at resonance. Moreover, such nantenna arrays have been fabricated on 4 inch $\times 4$ inch flexible polyethylene substrates using a stamp and repeat process. The master template used to create the nantennas consists of $\sim 10^{10}$ antenna elements on an 8 inch Si wafer.

Of recent note is the fabrication of a nanoscale YagiUda antenna. Conventional Yagi-Uda antennas (e.g., a TV antenna) are composed of a linear array of metal rods that serve as reflector (rear), one driven element (feed), and directors (in the direction of transmission or reception). The electromagnetic radiation emitted from the feed element induces currents in the other passive elements of the antenna array, resulting in directional, in phase emissions from all the elements in the array. Kadoya et al. fabricated a nanoscale Yagi-Uda antenna using $50 \mathrm{~nm}$ thick gold nanorods lithographically patterned on a glass substrate to perform strong directional control of red light $(662 \mathrm{~nm})$ that was injected at the feed element [40]. In this nanoscale version of the Yagi-Uda antenna, the size and location of the nanorods are chosen to produce constructive interference in one direction, and the conventional radiofrequency electronic circuit feed is simulated using the polarization dependence of a nanorod. The resonance wavelengths were found to be $610 \mathrm{~nm}, 655 \mathrm{~nm}$, and $770 \mathrm{~nm}$ for nanorods of length $75 \mathrm{~nm}$ (director), $106 \mathrm{~nm}$ (feed), and $125 \mathrm{~nm}$ (reflector), respectively. Kadoya et al. stated that the nanoscale YagiUda antenna could be fed in the near field by a collection of nanoparticles [40]. As reported by physicsworld.com, "this feat has now been achieved by Niek van Hulst and colleagues at the Institute for Photonic Sciences (ICFO) in Barcelona together with researchers at the Catalan Institute for Research and Advanced Studies (ICREA). They fabricated a number of nanoscale Yagi Uda antennas containing the tiny parasitic elements made from gold using lithography to etch the devices onto a glass substrate. The total length of individual antennas was $830 \mathrm{~nm}$ where individual feeds were just $145 \mathrm{~nm}$, each separated by $175 \mathrm{~nm}$." Further "by positioning the quantum dots close to the gold feed elements, the researchers were able to couple the quantum dots with the near field of the nanoantenna." [24]. This work is published in Science by Curto et al. [41].

The patch antenna structures require simpler fabrication than the 3D nanowire structures, due to the current and evolving nanolithography and nanofabrication techniques. The planar structures also allow for the fabrication of the rectifier discussed in this paper. The technological difficulty of producing arrays of nanometer gap junctions over areas of $\mathrm{cm}^{2}$ has recently been overcome by Gupta and Willis using selective atomic layer deposition [13]. Planar arrays of $\mathrm{Cu}$-vacuum- $\mathrm{Cu}$ tunnel junctions were produced on silicon wafers using conventional lithography techniques, followed by selective ALD to yield tunnel junctions of $\sim 1 \mathrm{~nm}$. See Figures 2 and 3.

In Appendix A, we outline the fabrication process for several realizations of rectenna devices that can be used to collect and rectify high frequency electromagnetic radiation. 


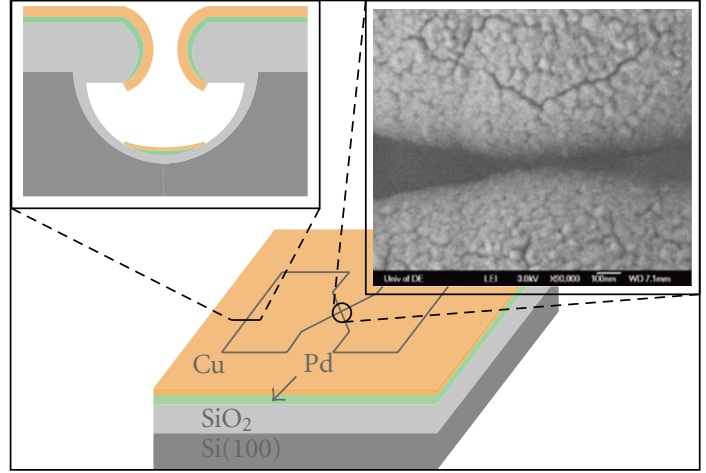

Figure 2: Schematic view of the device fabricated by ALD. The black region surrounding the device provides electrical isolation. A cross-sectional view is shown in the left inset. The right inset shows a scanning electron microscope image of the Pd seeded electrodes before $\mathrm{Cu}$ ALD [13].

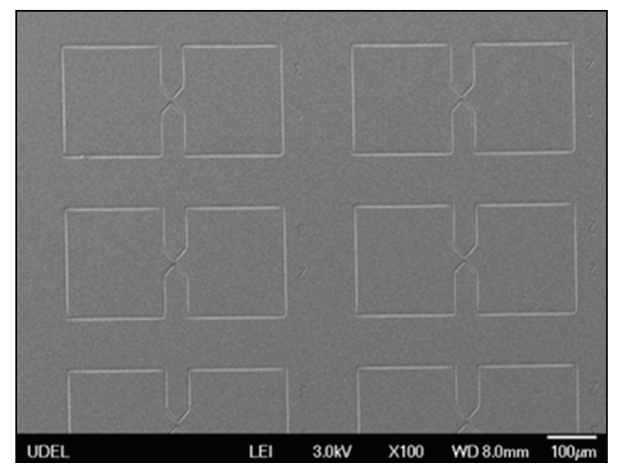

Figure 3: Scanning electron micrograph of an array of tunnel junctions fabricated using ALD [13].

\section{Review of Theoretical and Experimental Results on Rectification in Tunnel Junctions}

4.1. Mechanisms for Rectification in Tunnel Junctions. The I-V characteristics of a tunneling junction are determined by (1) the flux of electrons in given initial states incident on the barrier interface and their occupation probabilities that depend on temperature and field, (2) the available final states and their occupation probabilities, and (3) the shape of the tunnel barrier, which may be modified due to contact potentials, surface photovoltage effects, induced AC voltages due to laser irradiation, and so forth. Correspondingly, the current asymmetry (or rectification properties) at fixed gap width $s$ must originate from one or several possible causes discussed below, namely, material, geometrical, thermal asymmetry, and photo-stimulated changes in the electron flux distribution [42-44].

4.1.1. Material Asymmetry. This effect is expected to be most pronounced for metal/semiconductor tunnel junctions. A potential model illustrating material or work functioninduced asymmetry is sketched in Figure 4 for a planarplanar junction. When the electrodes have different work functions, the barrier shape will be asymmetric at zero bias. The barrier asymmetry is enhanced for forward and reverse bias. This results in different transmission probabilities for the same magnitude of bias voltage, as can be seen, for example, by inspection of the Wentzel-Kramers-Brillouin (WKB) transmission integral through a triangular barrier [8].

4.1.2. Thermal Asymmetry. This effect will take place when there is a temperature difference between the two electrodes and hence a different electron occupation of the states involved in tunneling; these states are generally from a narrow region centered around the Fermi level. As schematically indicated in Figure 5, the forward current would exceed the backward one even for a planar-planar junction of identical materials if the tip temperature were higher than the sample surface. In the limit of a high temperature, one recovers a situation analogous to ordinary thermionic emission. Thermally assisted field emission, that is, a combination of thermal and high-field effects, was in fact invoked as contributing to the mechanism of rectification in metal whisker diodes. It should be expected that, with laser irradiation, a differential heating of the tip and the base of the junction may occur causing a thermal asymmetry contribution to the tunneling current.

4.1.3. Geometrical Asymmetry. For nanometer gap distances, the nature of the tunneling phenomenon is such that the current passes predominantly through that sharp protrusion closest to the planar sample surface. In such conditions and even in the absence of any material asymmetry (e.g., W tip, W surface, and assuming no work function inhomogeneities), the shape of the tunnel barrier is asymmetric as a function of the applied bias field. This is due to the geometric asymmetry of the electrodes comprising the tunnel junctions. This effect is illustrated in Figure 6.

It is evident that the static electric field gradient is larger near the pointed apex of the tip than at the planar surface of the sample. This means that the vacuum tunnel barrier will tend to buckle inward or become thinner for forward bias and balloon outward or become thicker for reverse bias. The first observation of the geometrical asymmetry effect in an STM was observed by Feenstra et al. [45] and in the detailed study of rectification in an STM presented by Nguyen et al. [12]. In addition, Dagenais et al. [46] have experimentally verified that a geometrically asymmetric tunneling diode can be used to rectify radiation through the RF region. Based on their experiments, they envision that higher conversion efficiencies will be achieved at mid-IR frequencies. Most recently, Ward et al. [47] have shown both experimentally and theoretically that nonlinear tunneling conduction between gold electrodes separated by a subnanometer gap leads to optical rectification, producing a DC photocurrent when the gap is irradiated by a $785 \mathrm{~nm}$ laser source.

4.2. Theoretical Analysis of DC I-V Data for a Tunnel Junction. Before we present the experimental results and theoretical 


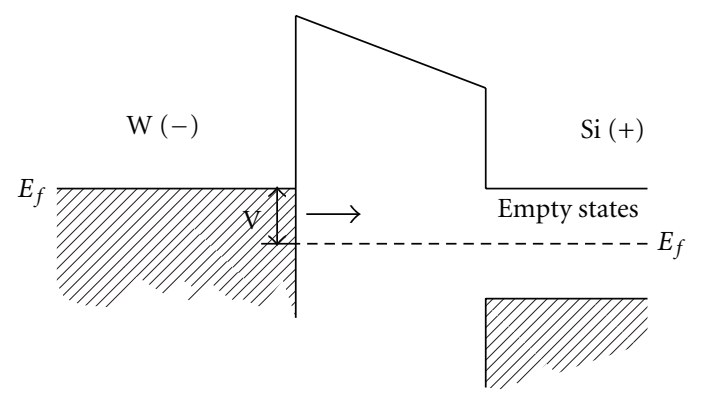

(a)

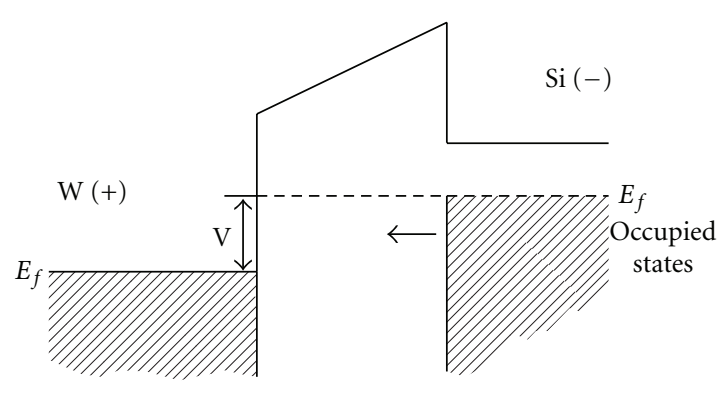

(b)

FIGURE 4: Schematic representation of asymmetric tunneling due to the material asymmetry of a junction such as tungsten/silicon (W/Si); (a) corresponds to forward bias $+\mathrm{V}$; (b) corresponds to reverse bias $-\mathrm{V}[12]$.

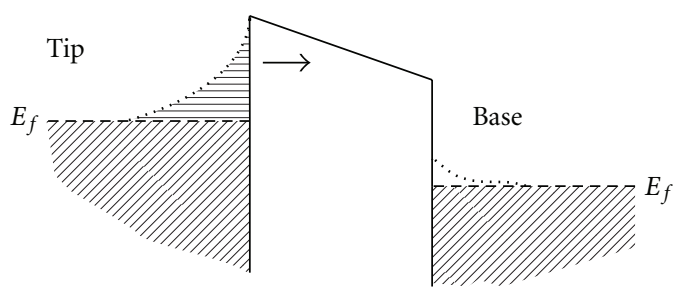

Figure 5: Thermal asymmetry of the tunnel current caused by the tip temperature greater than the base temperature for forward bias. The broader thermal spread of the electron distribution of the planar cathode should result in a higher forward bias current [12].

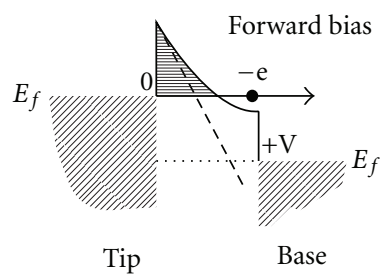

(a)

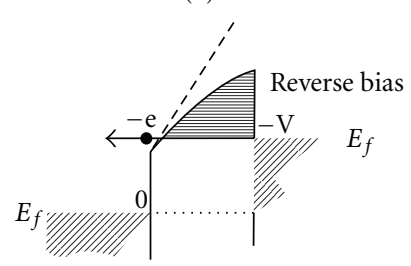

(b)

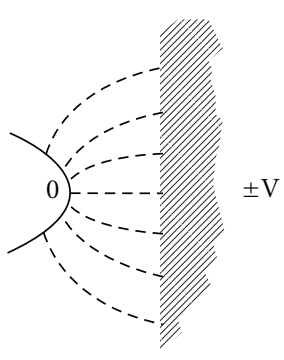

(c)
FIgURE 6: A tunnel barrier of a materially symmetric junction for a geometrically asymmetric junction shown in (c) exhibits a concave shape for forward bias (a) and convex shape for reverse bias (b) [12].

simulations to verify optical rectification, we present in this section a classical and quantum-mechanical methodologies for the analysis of the DC I-V characteristics to deconstruct the data in order to determine whether it exhibits optical rectification. Moreover, the analysis identifies some of the relevant experimental and theoretical quantities associated with this effect [21].

In the first part of this section, we present a classical approach for the analysis of the DC I-V data for a tunnel junction device (or any two-terminal device) that is generated from experiment or theoretical simulations in which there is a small AC voltage impressed. At the end of this section, we present the quantum corrected result due to photon-assisted tunneling applied to atomic-scale contacts, which is valid in the regime when $\hbar \Omega \gg e V_{\text {osc }}$.

Let us assume that the currents $I_{\text {stat }}$ actually induced by a static external bias $V_{\text {stat }}$ have been established. The current $I(t)$ induced by a potential $V(t)=V_{\text {stat }}+V_{\text {osc }} \cos (\Omega t)$ may be obtained from the Taylor expansion, yielding:

$$
I(t)=\sum_{n=0}^{\infty} \frac{1}{n !} \frac{d^{n} I_{\text {stat }}}{d V_{\text {stat }}^{n}}\left[V(t)-V_{\text {stat }}\right]^{n} .
$$

This expression assumes that the diode currents follow instantaneously the external potential in the limit where $\Omega \rightarrow 0$. This assumption will not hold when the period $T=2 \pi / \Omega$ of the external bias is comparable with the time taken by electrons to cross the junction, which is the case for optical frequencies.

Using the trigonometric relationship, $V_{\text {osc }}^{2} \cos ^{2}(\Omega t)=$ $\left(V_{\text {osc }}^{2} / 2\right)[1+\cos (2 \Omega t)]$, and similar expressions for higherorder terms, the diode current can be written in the following form:

$$
I(t)=\sum_{n=0}^{\infty} I_{n} \cos (n \Omega t)
$$

with

$$
\begin{aligned}
I_{0}= & I_{\text {stat }}+\frac{V_{\text {osc }}^{4}}{4} \frac{d^{2} I_{\text {stat }}}{d V_{\text {stat }}^{2}} \\
& +\frac{V_{\text {osc }}^{4}}{64} \frac{d^{4} I_{\text {stat }}}{d V_{\text {stat }}^{4}}+\frac{V_{\text {osc }}^{6}}{2304} \frac{d^{6} I_{\text {stat }}}{d V_{\text {stat }}^{6}}+\ldots, \\
I_{1}= & V_{\text {osc }} \frac{d I_{\text {stat }}}{d V_{\text {stat }}} \\
& +\frac{V_{\text {osc }}^{3}}{8} \frac{d^{3} I_{\text {stat }}}{d V_{\text {stat }}^{3}}+\frac{V_{\text {osc }}^{5}}{192} \frac{d^{5} I_{\text {stat }}}{d V_{\text {stat }}^{5}}+\ldots,
\end{aligned}
$$




$$
\begin{aligned}
I_{2}= & \frac{V_{\text {osc }}^{4}}{4} \frac{d^{2} I_{\text {stat }}}{d V_{\text {stat }}^{2}}+\frac{V_{\text {osc }}^{4}}{48} \frac{d^{4} I_{\text {stat }}}{d V_{\text {stat }}^{4}} \\
& +\frac{V_{\text {osc }}^{6}}{1536} \frac{d^{6} I_{\text {stat }}}{d V_{\text {stat }}^{6}}+\ldots,
\end{aligned}
$$

and so forth.

Hence, the DC component of the diode current is

$$
\langle I\rangle=I_{\text {stat }}+\frac{V_{\mathrm{osc}}^{2}}{4} \frac{d^{2} I_{\text {stat }}}{d V_{\text {stat }}^{2}},
$$

if we keep only the lowest-order term in $V_{\text {osc }}$. This term contains the information regarding the rectification of the optical signal by the device. The first term is the current produced by the applied bias and the second term is the additional rectified current due to the optical radiation. This expression was used by $\mathrm{Tu}$ et al. [48] to analyze their experimental data on rectification at microwave frequencies. This analysis was also used by Bragas et al. [49] to identify and separate out the contribution of the optically rectified current to the total measured DC current.

The energy gained, per unit of time, by the electrons that cross the junction is given by

$$
\langle P\rangle=\frac{\Omega}{2 \pi} \int_{0}^{2 \pi / \Omega} V(t) I(t) d t=V_{\text {stat }} I_{\text {stat }}+\frac{V_{\text {osc }} I_{1}}{2} .
$$

To lowest order, (4) reduces to,

$$
\langle P\rangle=V_{\text {stat }} I_{\text {stat }}+\frac{V_{\text {osc }}^{2}}{4} \frac{d I_{\text {stat }}}{d V_{\text {stat }}} .
$$

The rectified bias $V_{\text {rect }}$ corresponds to the static bias that would produce the same DC current as $V_{\text {osc }} \cos (\Omega t)$. Hence, one can determine $V_{\text {rect }}$ by the relation,

$$
V_{\text {rect }} \frac{d I_{\text {stat }}}{d V_{\text {stat }}}=\langle I\rangle-I_{\text {stat }} .
$$

Using (3), we obtain

$$
V_{\text {rect }}=\frac{V_{\text {osc }}^{2}}{4} \frac{d^{2} I_{\text {stat }} / d V_{\text {stat }}^{2}}{d I_{\text {stat }} / d V_{\text {stat }}}
$$

The quantum efficiency of the rectification process is defined, in this context, as the ratio of the DC current induced by the oscillating potential relative to the energy absorbed per unit time in the junction relative to the quantum limit of $e / \hbar \Omega$. Thus, the quantum efficiency is

$$
\eta_{\text {quant }}=\frac{\langle I\rangle-I_{\text {stat }}}{\langle P\rangle-V_{\text {stat }} I_{\text {stat }}} \frac{1}{e /(\hbar \Omega)} .
$$

Using (3) and (6), (8) becomes

$$
\eta_{\text {quant }}=\frac{1}{2} \frac{\hbar \Omega}{e} \frac{d^{2} I_{\text {stat }} / d V_{\text {stat }}^{2}}{d I_{\text {stat }} / d V_{\text {stat }}}=\left[\frac{2 V_{\text {rect }}}{V_{\text {osc }}}\right] \frac{\hbar \Omega}{e V_{\text {osc }}} .
$$

The classical impedance $R$ is,

$$
R=\frac{1}{\left(d I_{\text {stat }} / d V_{\text {stat }}\right)},
$$

and the classical responsivity $S$ (the DC current output per unit power input) is given by the expression:

$$
S=\frac{d^{2} I_{\text {stat }} / d V_{\text {stat }}^{2}}{d I_{\text {stat }} / d V_{\text {stat }}}
$$

The quantities $R$ and $S$ represent quantities that can be measured or deduced from model simulations of the junction and thus are of interest for characterizing the junction. Note that the rectified voltage is directly related to the responsivity,

$$
V_{\text {rect }}=\frac{V_{\text {osc }}^{2}}{4} S \text {. }
$$

A full-quantum mechanical treatment of radiation interaction with a nanoscale junction must take account of photon-assisted tunneling. For the special case of when $\hbar \Omega \gg e V_{\text {osc }}$ or $\left(e V_{\text {stat }} / \hbar \Omega\right) \rightarrow 0$, the DC current is given by the following expression [21]:

$$
\begin{gathered}
\langle I\rangle=I_{\text {stat }}+\frac{V_{\text {osc }}^{2}}{4}\left[\frac{I_{\text {stat }}\left(V_{\text {stat }}+\hbar \Omega / e\right)-2 I_{\text {stat }}\left(V_{\text {stat }}\right)}{(\hbar \Omega / e)^{2}}\right. \\
\left.+\frac{I_{\text {stat }}\left(V_{\text {stat }}-\hbar \Omega / e\right)}{(\hbar \Omega / e)^{2}}\right] .
\end{gathered}
$$

The relations established in this section therefore require that the classical theory is applicable when

$$
V_{\text {osc }}<\frac{\hbar \Omega}{e}<2\left[\frac{d^{2} I / d V_{\text {stat }}^{2}}{d I_{\text {stat }} / d V_{\text {stat }}}\right]^{-1} .
$$

Thus, this analysis is useful for infrared frequencies. Simulations by Mayer et al. $[20,21]$ have shown that deviations from the classical theory appear when considering frequencies in the visible domain due to the fact that electrons are promoted to higher energy levels because of the absorption of quanta of energy $\hbar \Omega$. This process is not accounted for by the classical analysis and increases the tunneling probabilities and the diode currents $[20,21]$.

4.3. Review of Experiments Verifying Rectification Mechanisms from the Microwave through the Visible Region. In this section we review some of the experiments verifying that an STM and other nanojunctions structures can act as antennas and rectifying devices for electromagnetic radiation from the microwave through the visible.

In a series of experiments by Kuk et al. [23, 50] used an STM consisting of a metal tip (Au) and a semiconductor sample (including Si $(111)-(7 \times 7))$ was illuminated with laser radiation below and above the semiconductor indirect band gaps, specifically, photon energies of $2.94 \mathrm{eV}, 1.96 \mathrm{eV}$, $1.17 \mathrm{eV}$, and $0.95 \mathrm{eV}$. The STMs were modified using a small lens to focus the laser beam to near its diffraction limit, yielding power densities up to $5000 \mathrm{~W} / \mathrm{cm}^{2}$ on the junction [51]. The resulting induced bias was measured laterally along the surface as light-induced excess current and voltage. For photon energies exceeding the band gap energy, surface photovoltages (SPVs) of about $300 \mathrm{mV}$ were induced across the gap independent of illumination intensity and frequency. 


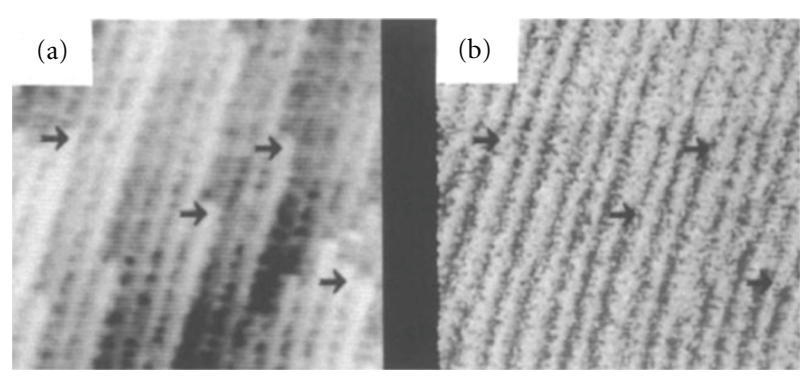

Figure 7: An STM topography of the $\operatorname{Au}(110)-(1 \times 2)(120 \times$ $120 \AA$ ) and a simultaneously taken light-induced current image [23].

For a photon energy of $0.95 \mathrm{eV}$, no surface photovoltage was detected. A small atomically scaled (laterally along the surface) varying DC signal of $3-5 \mathrm{mV}$ was also observed in the experiments. To explore the origin of this voltage and eliminate any induced SPVs, the semiconductor was replaced by an $\mathrm{Au}(110)-(1 \times 2)$ sample in the tunnel junction. In Figure 7, we show the topography of the $\mathrm{Au}$ surface and the corresponding photo-induced DC current image. The congruence between the two images is striking, with the spatial variation of the photocurrent corresponding exactly to the spacing of the troughs in the $\mathrm{Au}(110)$ surface structure. The AC-induced voltage is about $3 \mathrm{mV}$ and the authors suggest that this small signal is due to optical rectification associated with the geometric asymmetry of the junction. These striking results using an Au tip and collector demonstrate that the STM junction can absorb and rectify radiation corresponding to wavelengths shorter than $1.06 \mu \mathrm{m}$ in agreement with the experimental results of Nguyen et al. [12].

In order to qualitatively understand the above results for the semiconductor surface, we consider the basic processes involved in the production of a surface photovoltage. In the surface photovoltage effect in semiconductors, the irradiation of the surface by photons greater than the band gap produces electron-hole pairs. The SPV signal is a function of the excess minority carrier density injected into the surface space-charge region. The excess carrier density is in turn dependent on the incident light intensity, the optical absorption coefficient, and the minority carrier diffusion length. Thus, an accurate knowledge of the absorption coefficient versus wavelength is required for understanding SPV results. In Figure 8 we show the absorption coefficient of silicon as a function of wavelength from Green and Keevers [52]. The absorption coefficient is defined as the reciprocal of the distance for the intensity to decrease by a factor of $1 / \mathrm{e}$ of its initial value. Note that for energies above the band gap for $\mathrm{Si}$, the absorption coefficient is about $10^{4} / \mathrm{cm}$, whereas for the lower energies, the coefficients are at least 2 orders of magnitude smaller.

The observed SPV experimental results of Kuk et al. $[23,50]$ for semiconducting surfaces are consistent with the results in Figure 8. Moreover, given that the surface photovoltage is about $300 \mathrm{mV}$ for photon energies greater than the band gap energy (and, hence, are independent

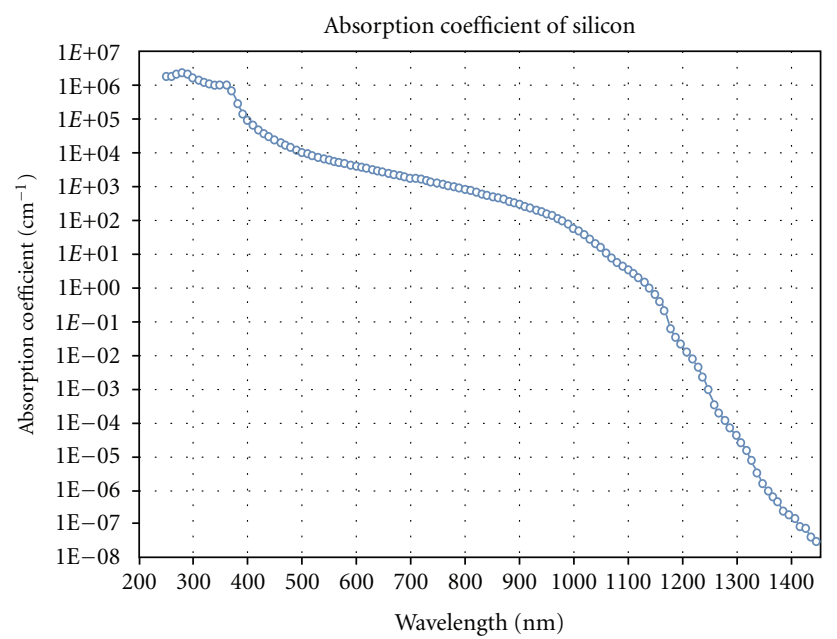

FIGURE 8: The absorption coefficient of silicon in $\mathrm{cm}^{-1}$ as a function of the wavelength. Silicon is an indirect bandgap semiconductor so there is a long tail in absorption out to long wavelengths. The data is graphed on a log scale. The drop in absorption at the band gap edge (around $1100 \mathrm{~nm}$ ) is much more rapid than that might appear due to the use of a log scale [24].

of wavelength in this range) suggests that the intensity is high enough to achieve saturation of the induced surface photovoltage.

Tu et al. [48] have experimentally verified that an STM junction can rectify radiation in the microwave region, which has led to the first direct, quantitative measurement of the rectification current due to single atoms and molecules. In their work, microwave of known amplitude and frequency irradiated the junction of a low temperature scanning tunneling microscope producing an electric field between the tip and an atom or molecule on the anode surface. It induced a DC signal that is spatially localized and exhibits chemical sensitivity at the atomic scale.

Bragas et al. [49] in 1998 used a laser with wavelength of $670 \mathrm{~nm}$ to irradiate an STM junction to determine the field enhancement as measured by optical rectification. A field enhancement factor between 1000 and 2000 was obtained for highly oriented pyrolytic graphite and between 300 and 600 for gold. Analysis of their data indicated optical rectification due to junction geometry as well as thermal asymmetry. The admixture of $d^{2} I_{\text {stat }} / d V_{\text {stat }}^{2}$ was determined to be significant only for p-polarized light and in phase with the intensity variation, consistent with the expected behavior for the rectified current. Their experiments indicate that visible light $(640 \mathrm{~nm})$ can be rectified using nm-sized tunnel junction devices.

Most recently, Ward et al. have shown both experimentally and theoretically that "nonlinear tunneling conduction" between gold electrodes separated by a subnanometer gap leads to optical rectification when the gap is irradiated by a $785 \mathrm{~nm}$ laser source, producing a DC current [47].

We provide additional details concerning the Nguyen et al. results [12], since their experiment (illustrated in Figure 9) in 1989 also determines the effective tunneling or transit time through an STM MVM junction barrier when 


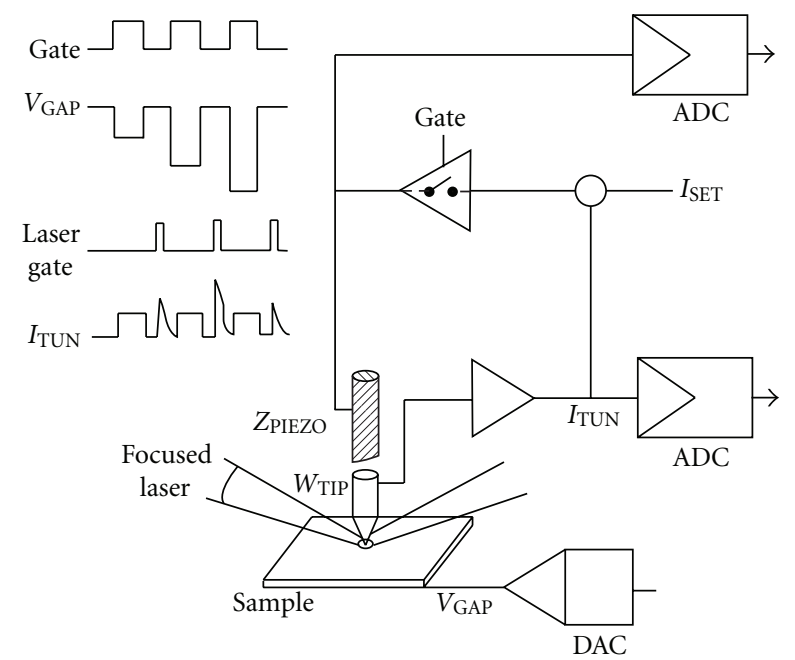

FIGURE 9: Schematic of laser irradiated STM junction rectification experiment from [12].

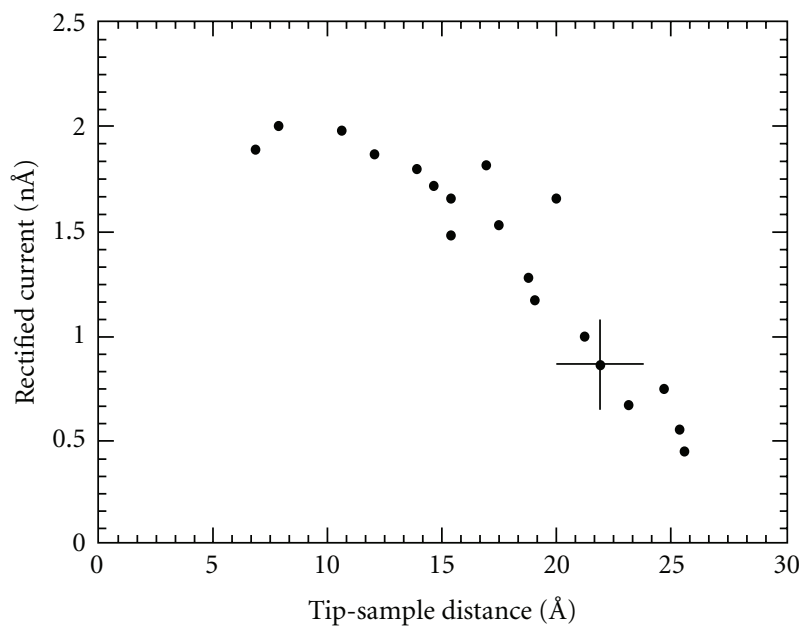

Figure 10: The measured rectified current is shown as a function of tip-sample distance for $\lambda_{\text {laser }}=1.06 \mu \mathrm{m}$. Cross-hair indicates estimated uncertainties in measurements [12].

irradiated with electromagnetic radiation. In the experiment a rectified DC current of about $1 \mathrm{nA}$ was measured as a function of tip-collector spacing (see Figure 10) for $\lambda_{\text {laser }}=$ $1.06 \mu \mathrm{m}$, a power of $5-20 \mathrm{~mW}$ and a beam diameter of $330 \mu \mathrm{m}$, incident on the junction, equivalent to about $20 \mathrm{~W} / \mathrm{cm}^{2}$. Note that in these experiments, SPV effects are not expected to be important at the wavelength used $(1.06 \mu \mathrm{m}$ or $1.17 \mathrm{eV})$. Note also, the results of Figure 10, done for a fixed frequency, can also be used to determine the limiting response time at that frequency [12]. Figure 10 demonstrates the optical rectification of IR frequencies for appropriate tunnel gap spacing $(d)$. If we assume an average tunneling velocity to be the Fermi velocity $\left(v_{f}\right)$, then $f_{\text {cutoff }}=$ $v_{f} / d$. This analysis predicts that a $1 \mathrm{~nm}$ gap is capable of rectifying radiation approaching the UV.

Weiss et al. [53] have recently conducted a set of experiments using the field emission and laser processing facilities

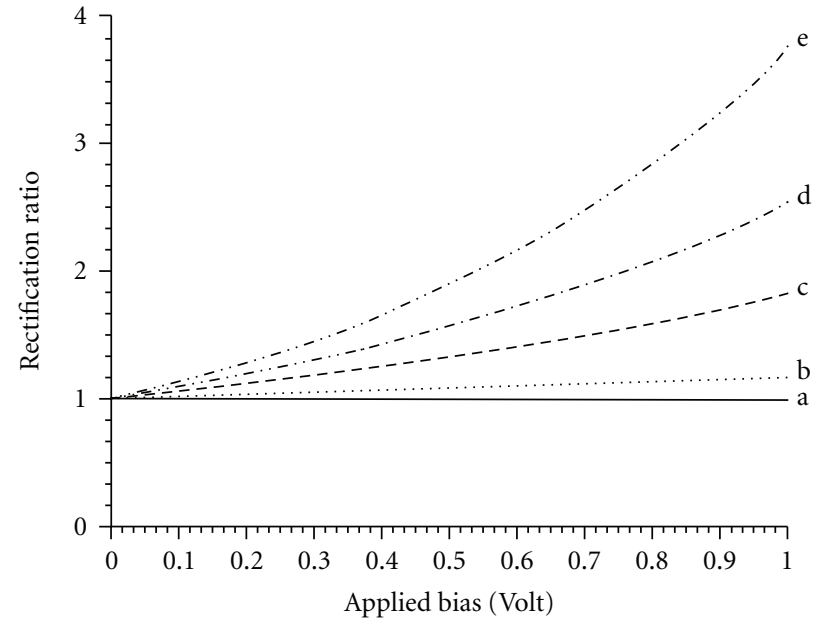

FIgURE 11: Effect of tip radius on the rectification ratios. The calculations are done for: $\rho=\infty$, that is, planar-planar junction, included as a reference (solid), (b) $\rho=10 \mathrm{~nm}$ (dotted), (c) $\rho=2 \mathrm{~nm}$ (dashed), (d) $\rho=1 \mathrm{~nm}$ (dot-dashed), (e) $\rho=0.5 \mathrm{~nm}$ (dot-dot dashed), and for the separation of $2 \mathrm{~nm}$ [25].

at the University of Puerto Rico's Rio Piedras campus. In these experiments a chemically etched tungsten tip was positioned near a molybdenum anode and irradiated with a HeNe laser $\left(\lambda=632 \mathrm{~nm}, P=1 \mathrm{~mW}\right.$ and $\left.d_{\text {spot }} \approx 1 \mathrm{~mm}\right)$. Initial results detected a rectified DC current of about $0.1 \mathrm{pA}$. An experiment on an open circuit configuration using a laser of increased power yielded an open circuit voltages of 10$70 \mathrm{mV}$. These results scaled with the results of Nguyen et al. who used a laser 1000 times the power of the recent experiments [12]. Although the rectified DC current is small, the anticipated device can contain an array with more than $10^{6}$ antenna elements (see Appendix A for a description of an initial prototype), thus producing a current of microamperes or larger. Additional experiments are anticipated using other laser frequencies $(1-10 \mu \mathrm{m})$, power levels $(10-1000 \mathrm{~mW})$, and more accurate determination of the power absorbed by the tip and will be reported in a subsequent publication. The Weiss et al. results are consistent with antenna theory and suggest that the laser produces a sufficient field for electron emission.

4.4. Review of Theoretical Work on Optical Rectification. Unlike a conventional planar MIM diode, the rectification process can be solely due (and/or primarily) to the geometrical asymmetry of the pointed nanowires/mCNTs tip. A razor-like edge (such as produced on a microstrip antenna, a cylindrical patch or other forms of patch antennas) can also provide the intrinsic geometric asymmetry necessary for the rectification process $[25,54]$. This geometric rectification is dramatically illustrated in Figure 11 where the rectification ratio of a $\mathrm{W}-\mathrm{W}$ junction (i.e., no material asymmetry) is compared for planar and pointed geometries [25].

Figure 11 shows that a planar geometry provides no rectification. The origin of the geometrical rectification process is illustrated in Figure 12. The rectification process 


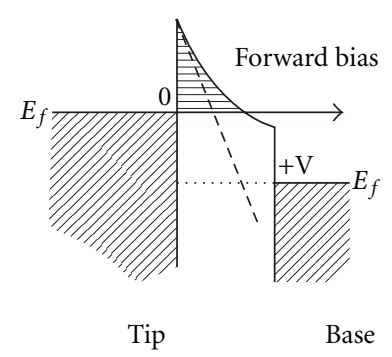

(a)

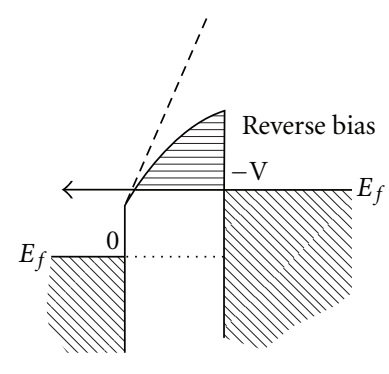

(b)

Figure 12: The (a) forward and (b) reverse asymmetric potential barriers for a geometrically asymmetric point-contact diode structure. Figure taken from [25].

can, of course, be enhanced by a temperature or material asymmetry and other geometric factors.

The early theoretical work on the tunneling characteristics of these junctions has usually relied on approximations in the shape of the barrier and in the tunneling probabilities, which were typically based on a one-dimensional model of the problem [8]. However, modern computational facilities make it possible to address this problem more rigorously using quantum-mechanical techniques including the threedimensional aspects of the problem and detailed atomic structure.

Lucas et al. [55] used a new formulation of elastic one-electron tunneling through three-dimensional (3D), nonseparable spatially localized barriers within the context of potential-scattering theory. To illustrate the principles of the method, it is applied to a model metal-vacuum-metal junction, which consisted of two parallel electrodes, one of which has a hemispherical protrusion. The electronic structure of each metal electrode is assumed to be freeelectron-like. The bias and multiple-image tunneling barriers for this model are constructed on the basis of classical electrostatics with a quantum correction at the metal surfaces. The barrier is composed of a planar separable part plus a nonseparable, localized perturbation due to the spherical boss. The exact, unperturbed, one-electron Green's function of the planar part is first obtained by numerical integration of the corresponding, effectively 1D Schrödinger equation. Then the localized boss potential is treated to all orders of perturbation by solving the Dyson equation for the full barrier Green's function, using a real-space discretization of the integral equation on a finite grid. Lucas et al. found that the current distribution peaks within a narrow angle around the boss axis, confirming earlier estimates based on the transfer-Hamiltonian formalism and in agreement with the observed atomic resolution of the microscope, when operating with atomic-size tips. Their Green's function method is applicable to several other problems of oneelectron tunneling through localized barriers and may be extended to incorporate such effects as the corrugation and the band structure of the electrodes.

Mayer et al. [17] presented a transfer-matrix analysis of this problem, which confirmed the conclusions achieved by Lucas et al. [25] and explored the dependence of the rectification properties of these systems on their physical and geometrical parameters. This analysis still relied on a quasistatic approximation, in which it is assumed that one can compare currents obtained for static values of the external bias. This approximation is valid in far infrared (frequency $f \rightarrow 0$ ) but must be replaced by a more exact approach in order to treat situations in which the time that electrons take to cross the junction is comparable with the period of the oscillating barrier.

In a subsequent paper Mayer et al. [18] extended his previous work by taking into account the time dependence of the external bias explicitly using the transfer-matrix approach and the time-dependent Schrödinger equation. As in their previous work, they focused on a system that consists of a cathode with a hemispherical protrusion and a flat anode. The geometrical configuration is illustrated in Figure 13.

Mayer et al. assume that the geometrically asymmetric tunnel junction in Figure 13 consisted of a cathode metal supporting a hemispherical protrusion with a height of $1 \mathrm{~nm}$, a radius of $0.5 \mathrm{~nm}$, and separation between the apex of the tip and the planar electrode of $1 \mathrm{~nm}$. Due to the external electromagnetic radiation of varying frequency and intensity, there is an impressed oscillating potential across the junction given by

$$
V_{\text {ext }}(t)=V_{\text {ext }} \cos \Omega t .
$$

In their simulations, $V_{\text {ext }}$ varies from $0.01 \mathrm{~V}$ to $1.0 \mathrm{~V}$ and frequencies that correspond to quanta of energy between $0.2 \mathrm{eV}(\lambda=6200 \mathrm{~nm})$ in the IR and $5 \mathrm{eV}(\lambda=248 \mathrm{~nm})$ in the UV. The rectification ratio $R=\langle I+\rangle /\langle I-\rangle$ that one obtains by taking the ratio between the mean values of the upward (forward), $\langle I+\rangle$, and downward (reverse), $\langle I-\rangle$, currents is represented in Figure 14. The values obtained at low frequency $\Omega \rightarrow 0$ agree with those obtained in their quasi-static analysis [17]. Because of the photon-absorption processes, the rectification ratio $R$ first increases with $\Omega$ before decreasing at higher frequencies. The intermediate region proves that the rectification of optical frequencies can be achieved by the device, which agrees with early conclusions reached by Sullivan et al. [14]. In our quasi-static analysis, they predict a cutoff of the rectification for a photon energy around $4 \mathrm{eV}$ (radiation with wavelength of $300 \mathrm{~nm}$ ) in ultraviolet because it was estimated that the field would then reverse before the electrons can cross the junction. This oscillating-barrier analysis shows indeed a significant decrease in the rectification at that frequency.

The results of Mayer also indicate that there is a time shift between the peak intensities of the oscillating barrier and 


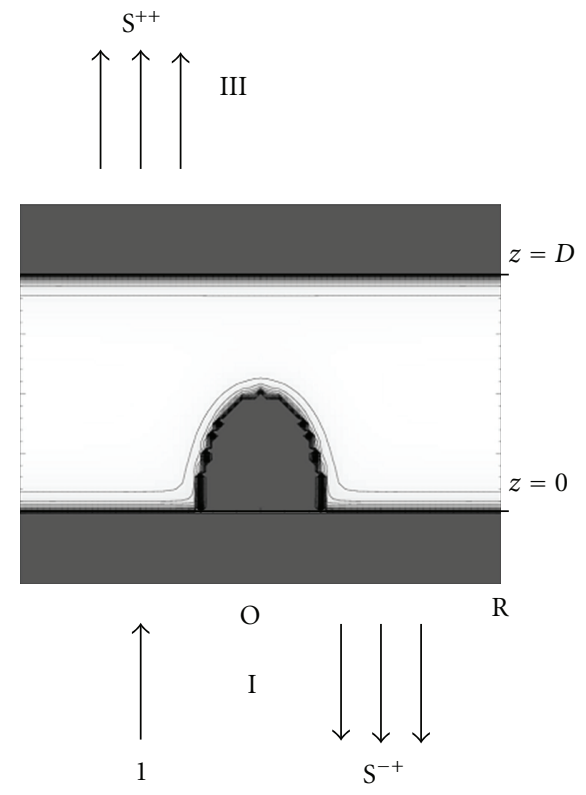

Figure 13: Schematic of theoretical model with protruding tip. $\mathrm{D}$ is the electrode separation. The arrows represent the incident and scattered waves used in the transfer-matrix simulation. The arrows in regions I and III symbolize scattering solutions, with a single incident state in region I and the corresponding reflected and transmitted states denoted by $\mathrm{S}^{-+}$and $\mathrm{S}^{++}$, respectively [18].

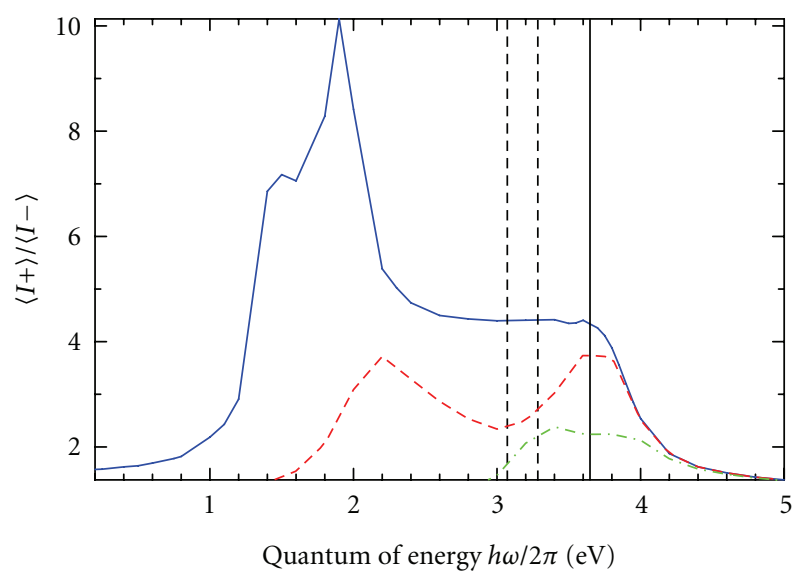

Figure 14: (Color online) Rectification ratio as obtained for a geometrically asymmetric junction subject to an external bias $V_{\text {ext }}(t)=V_{\text {ext }} \cos (\Omega t)$ with $V_{\text {ext }}=1$ (solid line), 0.1 (dashed d $\left._{-}\right)$ and $0.01 \mathrm{~V}$ (dot-dashed line). The quantum of energy $\hbar \Omega$ ranges between 0.2 and $5 \mathrm{eV}$. The vertical lines indicate the height of the surface barrier (as measured from the Fermi level of the emitting metal) when $V_{\text {ext }}(t)=1$ (dashed line, left), -1 (dashed line, right), and $0 \mathrm{~V}$ (solid Line) [18].

those of the diode currents due to the time that electrons take to cross the junction, which increases with the frequency of the oscillating barrier [18]. He attributes this to the fact that the time that electrons take to cross the junction contains a part that is intrinsic to the dynamics of these electrons, in particular, to the tunneling process that takes place in

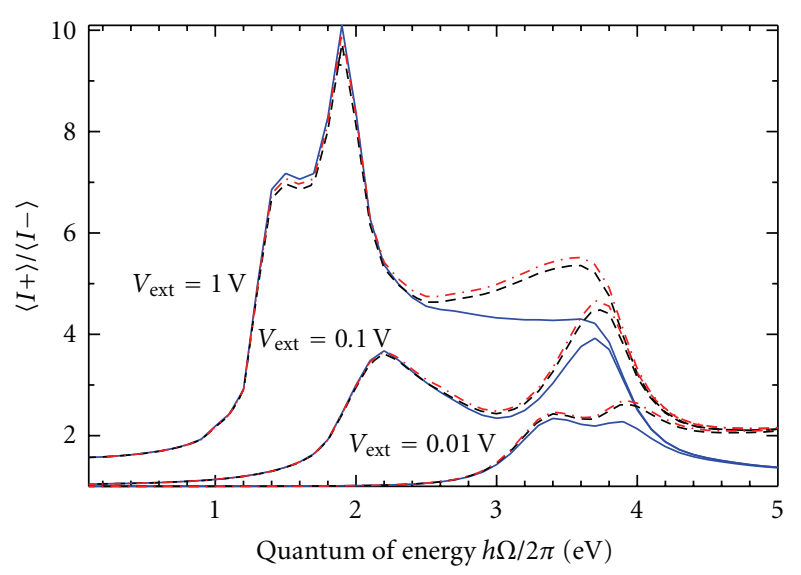

Figure 15: Rectification ratio of a junction made of tungsten and subject to an external bias $V_{\text {ext }}(t)=V_{\text {ext }} \cos (\Omega t)$, with $V_{\text {ext }}=1,0.1$ and $0.01 \mathrm{~V}$ (downwards as indicated). The solid, dashed and dotdashed lines indicate results achieved respectively with $\varepsilon(\Omega)=-\infty$, $\varepsilon(\Omega)=1-\Omega_{p}^{2} / \Omega^{2}$, and $\varepsilon(\Omega)=1-\Omega_{p}^{2} /\left(\Omega^{2}+i \Omega / \tau\right)$ as models for the dielectric function of the tip [19].

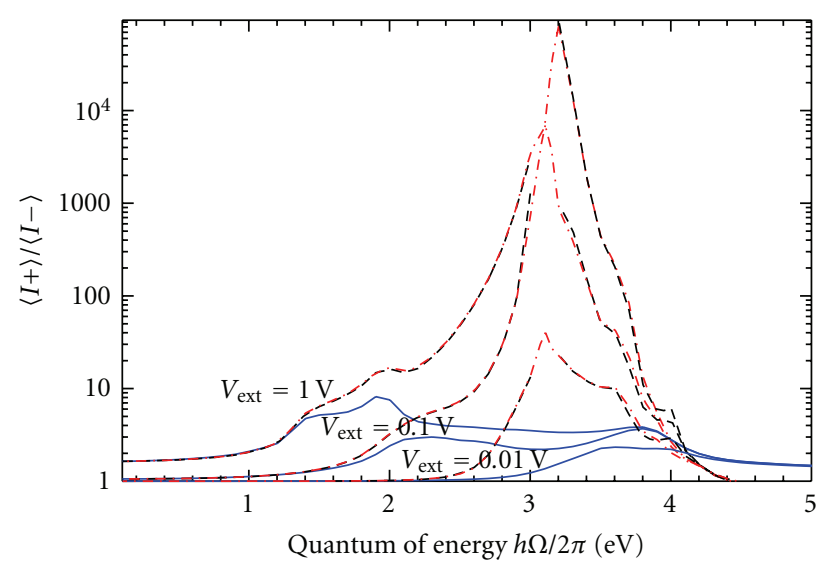

FIGURE 16: Rectification ratio of a junction made of silver and subject to an external bias $V_{\text {ext }}(t)=V_{\text {ext }} \cos (\Omega t)$, with $V_{\text {ext }}=1,0.1$ and $0.01 \mathrm{~V}$ (downwards, as indicated). The solid, dashed and dotdashed lines indicate results indicate results achieved respectively with $\varepsilon(\Omega)=-\infty, \varepsilon(\Omega)=1-\Omega_{p}^{2} / \Omega^{2}$, and $\varepsilon(\Omega)=1-\Omega_{p}^{2} /\left(\Omega^{2}+\right.$ $i \Omega / \tau$ ) as models for the dielectric function of the tip. Results obtained at the $3.1 \mathrm{eV}$ resonance energy with $\varepsilon(\Omega)=1-\Omega_{p}^{2} / \Omega^{2}$ as model for the dielectric function of the tip are not represented [19].

the classically forbidden part of the potential barrier. Mayer notes that detailed analysis of these time shifts may thus lead to an estimation of this tunneling time, a problem they do not address in this publication.

In order to assess the importance of the deposition of noble metals (known to have plasmonic resonances) on the field enhancement and rectification properties of a model tunneling junction, Mayer and Cutler performed 3D quantum mechanical computer simulations of optically irradiated MVM tunnel junctions using Ag and W tips [19]. They predict an enhanced rectification and current output due to 
the surface plasmonic resonances in $\mathrm{Ag}$ at $\sim 3 \mathrm{eV}$, corresponding to the energetic green portion of the visible spectrum. In particular, they investigate the influence of the dielectric function of the material considered for the tip. They compare the rectification achieved when tungsten or silver is used as material for the tip. Silver was considered because of the presence of plasmons in the range of frequencies at which the junction is the most efficient for the rectification of external signals. This study aimed at determining the role of these plasmons and more generally of the frequencydependence of the dielectric function on the rectification properties of the junction. In Figures 15 and 16, we illustrate the results of their simulations. Compared to tungsten, the power gained by the electrons (that cross the device) and the rectification ratio of this device are enhanced by several orders of magnitude at frequencies that correspond to a resonant polarization of the tip. These results suggest that the dependence of the plasmon frequencies on both the material and the geometry of the tip could be used to control the frequency at which the junction is the most efficient for the rectification of external signals. It is also important to note that there is a significant contribution of multiphoton processes (especially for $\hbar \Omega \ll\left|e V_{\text {ext }}\right|$ ), which is most pronounced when a polarization resonance occurs. This effect opens the possibility to build devices for the selective detection of radiation in the infrared or visible domain or for a more efficient rectification and conversion of their energy. It is expected that deposition of a thin layer of other noble metals on an underlying antenna structure such as tungsten, molybdenum, or aluminum should yield similar results.

In a 2009 publication, Mayer et al. [20] used a transfermatrix methodology to study the rectification properties of geometrically asymmetric metal-vacuum-metal junctions in which one of the metals is flat while the other is extended by a tip, whose aspect ratio is variable. They studied in particular the efficiency with which the energy of incident radiation is converted by this device. The radiation considered was either monochromatic (with typical frequencies in the infrared and visible domains) or characterized by a full range of frequencies in order to simulate the incidence of a focused beam of solar radiation. The results show that the efficiency of the energy conversion increases with the aspect ratio of the tip and that quantum efficiencies as high as $25 \%$ can be achieved with the system considered in this work. This dependence of the conversion efficiencies on the height of the tip suggests that larger protrusions should perform still better. They also observed a significant enhancement of the energy conversion at frequencies that correspond to a resonant polarization of the tip. The dependence of these resonance frequencies on the shape and on the material used for the tip therefore gives the possibility of controlling the frequency at which the device is especially efficient for the rectification of external radiation. It was finally shown that reducing the work function of the metallic elements increases the performance of this device. For practical applications, one may consider two-dimensional arrays in which the systems considered in this work would be placed with a typical spacing of $10 \mu \mathrm{m}$ between adjacent protrusions. The currents and energies achieved per square meter correspond in this case to those achieved for a single tip times a typical factor of $10^{10}$. Considering the possibility of a further focusing of the incident radiation, the results achieved in this work are quite significant and justify the consideration of these systems. They demonstrate that the rectification of radiation with typical frequencies in the infrared and optical domains can be achieved by using geometrically asymmetric metal-vacuum-metal junctions of the type presented in this work. These results finally provide a more quantitative analysis of the efficiency with which the energy of incident radiation is converted by the device.

In recent work by Mayer et al. [21], they investigate the frequency limits of a quasi-static classical analysis of geometrically asymmetric metal-vacuum-metal junctions in which a combination of static and oscillating biases is established between a cathode (i.e., extended by a hemispherical protrusion and a flat anode) as compared to a rigorous quantum mechanical transfer-matrix treatment. The static currentvoltage characteristics of this device are established using a transfer-matrix methodology. The rectification properties of the device are however analyzed in the framework of a classical model that is based on the Taylor-expansion of static current-voltage data (see Section 4.2). This enables the impedance and the classical responsivity of the device to be established. They then investigate how the impedance and the classical responsivity of this junction are affected by the work function of the materials, the gap spacing between the cathode and the anode, and the aspect ratio of the protrusion. Comparisons are made between the responsivity obtained using a classical approach with the quantum responsivity (determined from the currents produced in an oscillating barrier using a quantum mechanical treatment). The results for the responsivity and mean diode current using the quantum transfer matrix technique are comparable with the classical analysis only in the low frequency limit when $\Omega \rightarrow 0$. For a junction shown in Figure 13 (work function of the electrodes of $4.5 \mathrm{eV}$, with a height of $1 \mathrm{~nm}$, a radius of $0.5 \mathrm{~nm}$, and the separation between the apex of the tip and the planar electrode of $1 \mathrm{~nm}$, and $V_{\text {osc }}=$ $0.1 \mathrm{~V})$, the classical and the quantum mechanical results are in reasonable agreement when the photon energy is less than $0.4 \mathrm{eV}(\lambda=3100 \mathrm{~nm})$. The mean diode current obtained from the exact quantum mechanical scattering treatment is higher than that predicted from the classically predicted result. Significant deviations (a factor of 2) occur at photon energies of about $1 \mathrm{eV}(\lambda=1240 \mathrm{~nm})$ and increase as photon energies increase. These differences can be attributed to the fact that electrons are promoted to higher energy levels because of the absorption of energy quanta, increasing their tunneling probability. Deviations between the classical and quantum results should be more dramatic for lower work function materials. The enhanced diode currents due to the photon-absorption processes also result in effective responsivities that are higher than those predicted from a classical analysis. For example, the responsivities achieved at zero static bias (for a device with a work function of $1.5 \mathrm{eV}$, with a separation of $1 \mathrm{~nm}$ between the apex of the emitter and the anode) range from $0.72 \mathrm{~V}^{-1}$ in the far infrared (classical result) up to $35.8 \mathrm{~V}^{-1}$ for $\hbar \Omega=2 \mathrm{eV}$ 


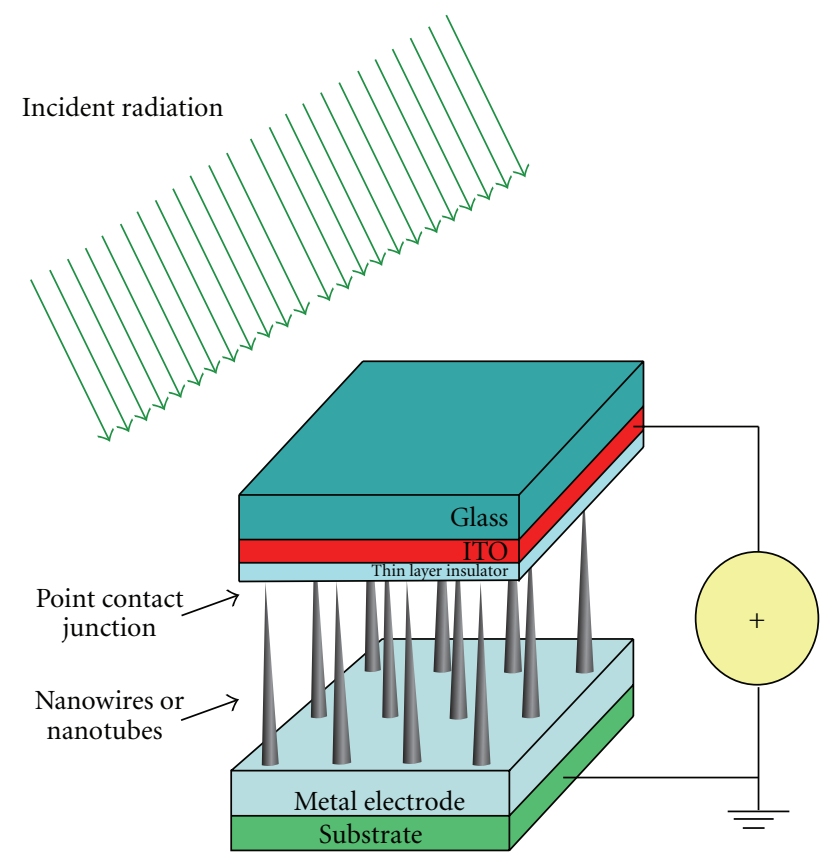

Figure 17: Schematic diagram of the proposed nanowire/CNT based IR sensor. The sharp metallic nanowires/CNTs act as the receiving antennae and the rectifying diodes.

(620 $\mathrm{nm}$ visible). Moreover, Mayer et al. emphasize that the classical analysis relies on current-voltage data for the ideal static case in which the dielectric constant of the materials $\varepsilon=-\infty$. This classical approach determines the current and the power in the dynamic case (under irradiation) without taking into account of the frequency-dependence of $\varepsilon$. With tungsten, the effects of a variation of $\varepsilon$ can be neglected for the range of frequencies throughout the optical region since the plasma energy for tungsten is about $22.8 \mathrm{eV}$. However, for silver, there is a surface plasmon energy at $3.6 \mathrm{eV}$. For $\mathrm{Ag}$ and other materials, such as $\mathrm{Cu}$, the frequency dependence of the dielectric constant must be taken into account and the quantum-mechanical treatment using a scattering theoretical approach should be used. This work provides additional insight for the development of an efficient device that could be used for the energy-conversion of infrared and optical radiations. Based upon the results summarized above, it is argued that an accurate treatment of nanoscale tunnel junctions operating in the near IR and visible requires a quantum mechanical treatment such as the approach used by Mayer et al. [17-21].

\section{Operational Designs for an Optical Rectenna}

This paper presents a new approach to optical rectifiers, which, for example, can be used in devices for harvesting and rectifying radiation from the infrared (IR) to the visible.

One realization of a rectenna based on this novel approach consists of nanowires/mCNTs on planar substrates with a point contact-like rectifying junction as shown in Figure 17. The device uses wavelength dependent sized

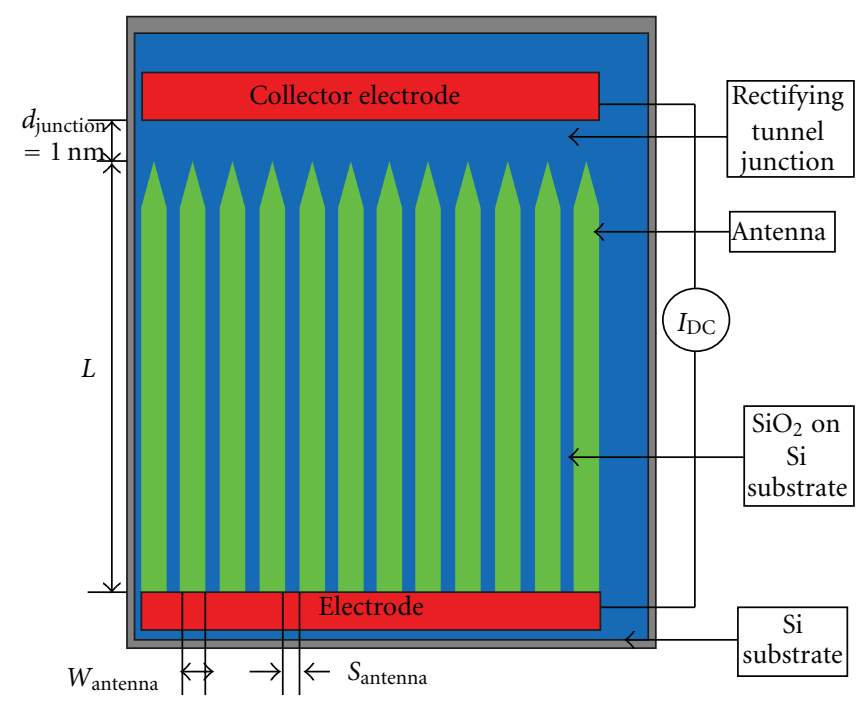

(a)

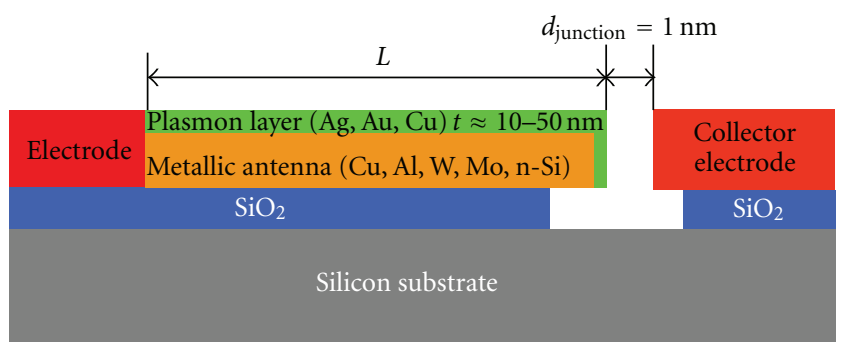

(b)

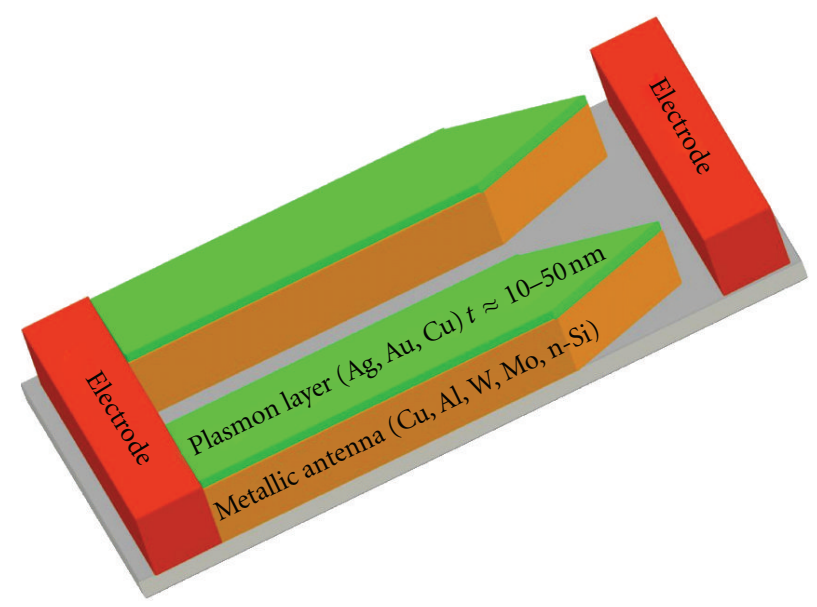

(c)

FIGURE 18: Schematic of proposed rectenna device. (a) Top view, (b) side view, and (c) 3D perspective.

vertical arrays of nanostructures with point contact-like junctions. The nanowire or mCNT forms a MVM or MIM junction barrier. Charge transport through the barrier is via tunneling. The problem of limited frequency response of conventional planar MIM diodes (limited by parasitic capacitance effects) is overcome by the use of these pointcontact nanowires/mCNTs, and their inherent fast response time (i.e. up to a femtosecond) [16]. 


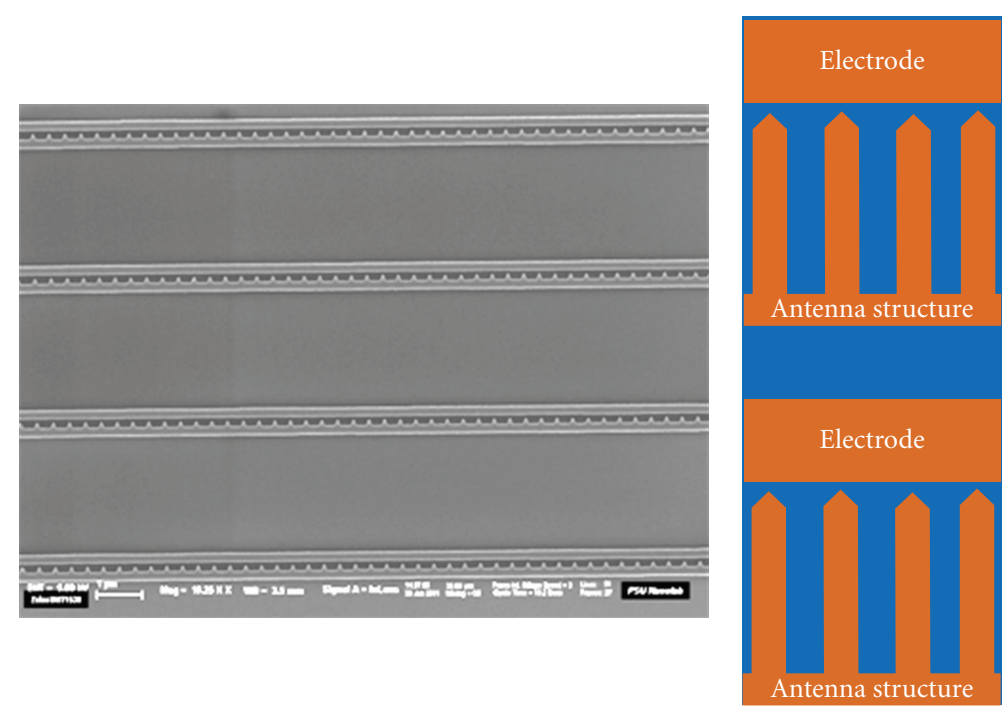

(a)

(b)

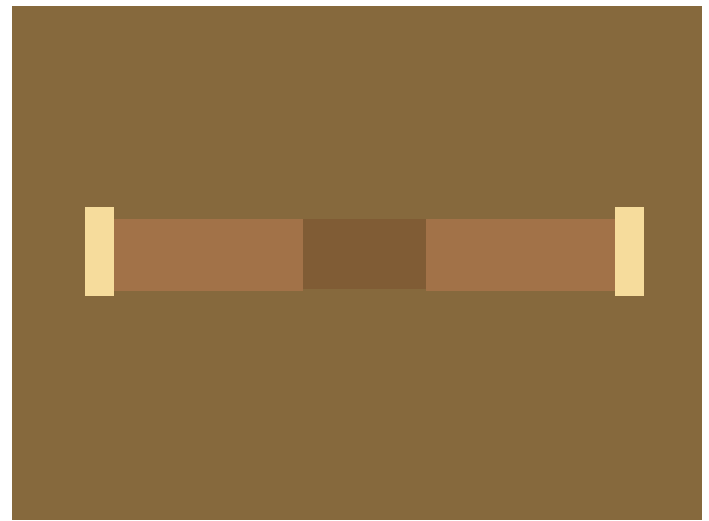

(c)

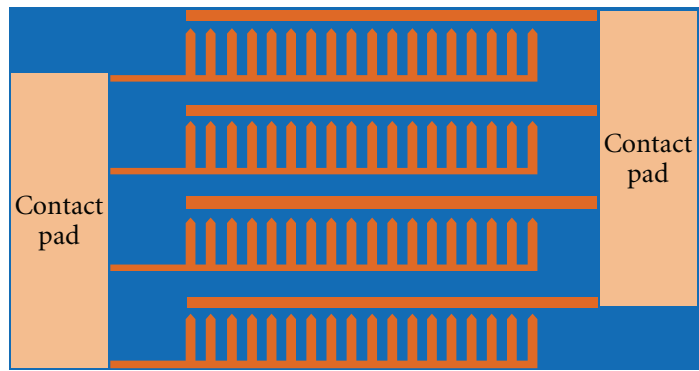

(d)

FIGURE 19: Fabricated rectenna device is shown on the left and a schematic diagram is on the right showing (a) top view SEM image (b) schematic of same; (c) optical image of device (darkerspacing between electrodes b) top view of same and (c) an array of rectennas connected to contact pads.

In Appendix A, fabrication processes are outlined for several other realizations of rectenna devices (e.g., thin wire with triangular tip, triangular patch or half-bow tie, rectangular patch) that can be used to collect and rectify high frequency electromagnetic radiation. It is important to note that these device structures can be fabricated using current nanofabrication techniques. Such facilities are available in the Penn State Nanofabrication Facility on the University Park Campus in Pennsylvania (http://www.nnin.org/nnin_psu.html).

\section{Summary and Future Work}

In summary, it has been argued theoretically and demonstrated by initial experiments the feasibility of this device as a viable IR sensor. It is also both implicit and explicit that this rectenna device will scale across and beyond the IR spectrum, since the response time of the diode is well into the visible regime.

It is clear from the review presented that there is a need for a series of targeted experiments and theoretical work to understand more rigorously the interaction of electromagnetic radiation with nanoantennas and other nanostructures $[31-33,56]$. At visible light frequencies the classical skin depth in metals is about $30 \mathrm{~nm}$ and metals become transparent in the UV. New simulations of these metal nanostructures are needed to determine their response and properties under electromagnetic illumination. Moreover, continuing experiments are needed to develop new materials and characterization of nanojunctions as a function of irradiance, wavelength, and polarization, and other properties to optimize the operating rectenna. In addition, important outstanding problems are the determination of the theoretical limit of the efficiency of these devices, and the solution of the practical materials and structural issues that are needed for optimization of the rectifier. In Appendix B, simple model estimations of the efficiencies of two of the devices discussed in this paper are presented.

It is not inappropriate to note that at this point that there have been suggestions that electron emission into fluids can obviate the need for vacuum in solar devices for 

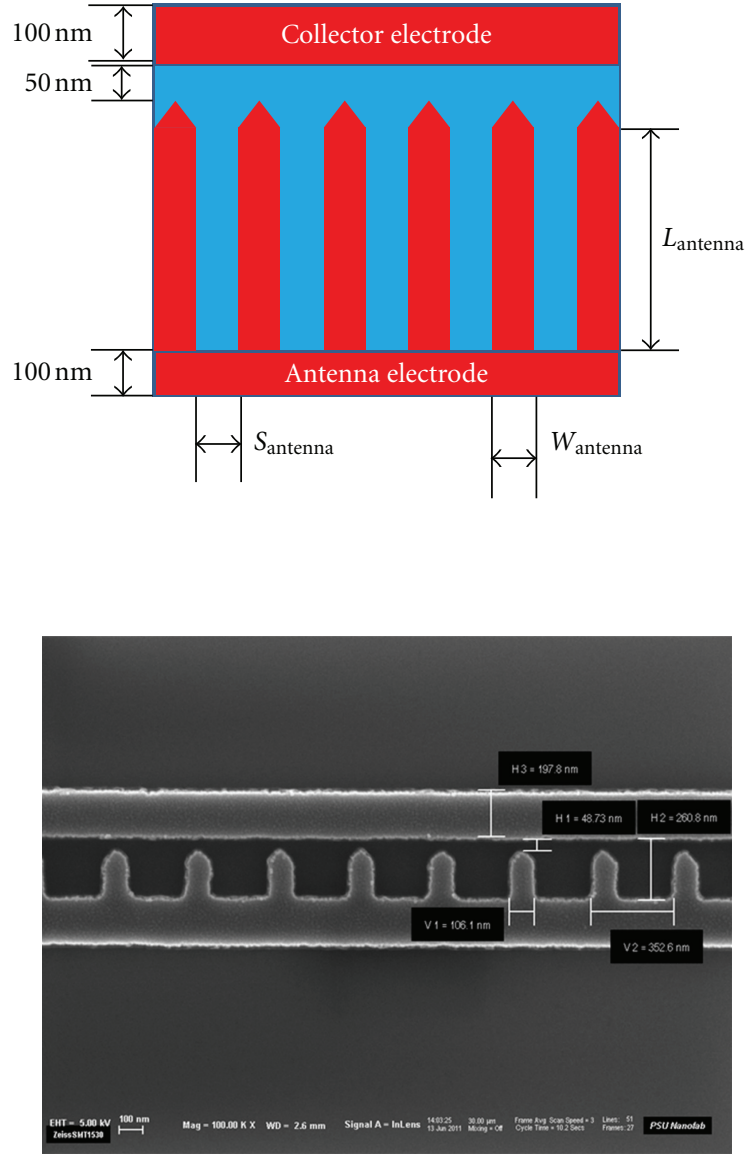

FIgUre 20: Schematic (top) and SEM image (bottom) of the top view for the columnar antenna with triangular tip.

energy conversion. Schmidt et al. of Rice University private communication have reported initial results of illuminating MWCNTs in arrays with various forms of simulated solar radiations and observing measurable currents. Although these results are very preliminary, they are sufficiently provocative to warrant further investigations, but in a later period of work. A most relevant source of useful studies of electron current emitted into fluids is given in the paper by Gomer, whose results indicate that fluids can lead to a significant reduction of the effective workfunction (see the review article: R. Gomer, Rep. Prog. Phys. 53 (1990) 917). Schmidt et al. also point out the complications such as ionic currents, heating effects within the dielectric layer, space charge, and other processes connected with charge transport.

\section{Appendices}

\section{A. Rectenna Fabrication Process}

We briefly describe our initial design and fabrication of the arrays of optical rectenna prototype. This is intended to demonstrate the feasibility of the fabrication process using established or conventional nanofabrication methods.

Two patch antenna structures, that is, triangle (bowtie) and "thin wire" terminated with a triangular tip, are
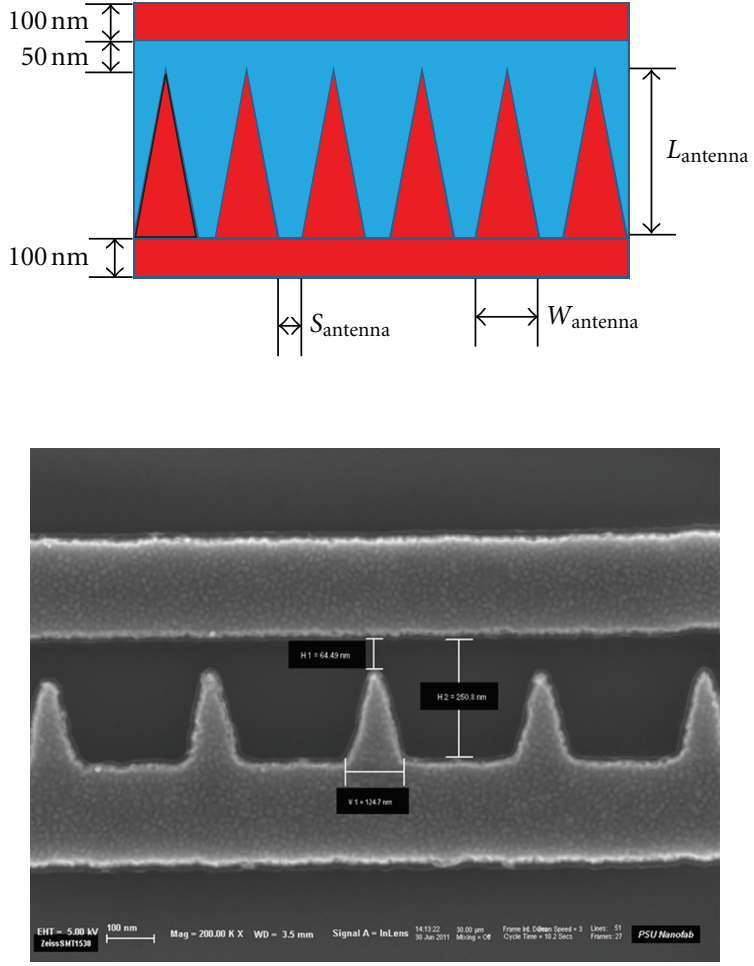

FIGURE 21: Schematic (top) and SEM image (bottom) of the top view for the triangular antenna.

illustrated in Figures 20 and 21. The antenna arrays have recently been fabricated by conventional lithography and ebeam techniques. The technological difficulty of producing arrays of nanometer gap junctions over areas of $\mathrm{cm}^{2}$ has recently been overcome by Gupta and Willis using atomic layer deposition (ALD) [13]. Planar arrays of Cu-vacuum$\mathrm{Cu}$ tunnel junctions were produced on silicon wafers using conventional lithography techniques, followed by ALD to yield tunnel junctions of $\sim 1 \mathrm{~nm}$. See Figures 2 and 3 .

Figures 18(a)-18(c) depict an idealized thin wire structure. The antennas are fabricated using e-beam lithography written on top of conventional lithography for the electrode structures. The plasmon layer is deposited using selected area ALD of copper which selectively deposits $\mathrm{Cu}$ only on the antenna and electrodes. The self-limiting growth process creates a gap between the collector electrode and antenna tip of $\sim 1 \mathrm{~nm}$.

Figure 19 depicts an array of rectennas with electrical connections. On the left are the optical images of the fabricated module and on the right are the schematic diagrams of the device.

Figures 19, 20, and 21 show the SEM images of a fabricated device module before ALD. The active sensor area $(\sim 400 \times 200 \mu \mathrm{m})$ contains about 10,000 rectennas. The dimensions of the antennas of each of the modules are designed to optimize absorption in the red and IR.

Electrical and optical characterization of these devices will be done before and after ALD processing and will be reported in a subsequent publication. Preliminary images 
of the devices after selective ALD at the University of Connecticut show that the $\mathrm{Cu}$ successfully grew on the Pd tips and narrowed the gap. Also, no growth was observed on the contact pad ( $\mathrm{Al})$ and surrounding $\mathrm{SiO}_{2}$ areas, as expected [57].

\section{B. Estimation of the Efficiency of Rectenna Devices}

One important consideration for future applications will be a determination of the efficiency of the rectifier. In this appendix we use a simple standard definition for the efficiency given by,

$$
\eta=\frac{P_{\text {out }}}{P_{\text {in }}}
$$

where $P_{\text {out }}$ is the output power and $P_{\text {in }}$ is the input power to the device, to estimate the efficiencies of two of the devices described earlier.

This quantity is frequency, material, and geometry dependent for all devices including photovoltaics and rectennas (that may also be used as energy harvesting devices). For photovoltaics, the DC power output is also strongly bandgap and resistivity dependent. By contrast, in the proposed rectenna devices, the efficiency depends on both the controllable geometry as well as the material parameters (e.g., electrical and optical constants) with no bandgap dependence for metallic tips. Distinct advantages of the metallic whisker (nanowire) rectenna are the demonstrated fast response up to the green part of the spectrum, as well as the low resistivity, which reduces the heat losses in the system. For energy harvesting devices, these facts suggest an increased efficiency of the point-contact metallic nanowires over photovoltaic devices using standard MOM or Schottky diodes. The theoretical efficiency requires the determination of output DC power. This can be calculated in the solar spectrum range of frequencies using the model of Sullivan [16] and, more exactly,using the quantum mechanical formalism of Mayer et al. [20].

We describe briefly a model calculation for the efficiency of a nanowire rectenna irradiated with the equivalent of solar radiation. The output power is given by: $P_{\text {out }}=J A_{e} V$, where $J$ is the field emitted current density, $A_{s}$ is the emitting area, and $V$ is the DC rectified voltage developed between the tip and base. The effective input power to the rectenna for the solar intensity is given by: $P_{\mathrm{in}}=I_{s} A_{b}$, where $I_{s}$ is the solar intensity of $0.1 \mathrm{~W} / \mathrm{cm}^{2}$ and $A_{b}$ is the area of the beam intercepted by the nanowire. Therefore,

$$
\eta=\frac{P_{\text {out }}}{P_{\text {in }}}=\frac{J V A_{e}}{I_{s} A_{b}} .
$$

To estimate these quantities, we use the conventional FowlerNordheim equation:

$$
J=\frac{1,54 \times 10^{-6} F^{2}}{\varphi \operatorname{Exp}\left(-6.83 \times 10^{7} \varphi^{3 / 2} / F\right)} .
$$

Here $F$ is the local field at the tip in $\mathrm{V} / \mathrm{cm}$ and $\varphi$ is the workfunction in $\mathrm{eV}$, with $J$ having units of $\mathrm{A} / \mathrm{cm}^{2}$.
The field values are obtained from the work of Sullivan. The corresponding values of $V$ and $F$ are obtained from Figure 15 in the same reference. The area of the emitting tip is estimated from a standard model calculation for field emitters. $I_{s}$ is the solar irradiance equal to $0.1 \mathrm{~W} / \mathrm{cm}^{2}$ and $A_{b}$ is approximately one-half the cylindrical surface area of the rectenna. The efficiency is calculated assuming the local electric fields at the tip, $F=1-5 \times 10^{7} \mathrm{~V} / \mathrm{cm}$, obtained from the work of Sullivan et al. $[14,16]$. With $F$ determined, $V$ is known. For a tip of radius of $2 \mathrm{~nm}$ and field values consistent with Sullivan et al. $\left(\sim 3-5 \times 10^{7} \mathrm{~V} / \mathrm{cm}\right), \eta \geq 50 \%$

It is seen that the predicted efficiencies may be equal or exceed efficiencies of competitive technologies. An important conclusion from this model calculation is that the efficiency can be controlled by judicious choices of material (e.g., the work function, $\varphi$ and geometric parameters of the tip. Although these calculations are based on radiation in the IR region of the solar spectrum (i.e., $\lambda \sim 1 \mu \mathrm{m}$ ), a more complete calculation will involve extending the wavelength into the visible region of the electromagnetic spectrum. In the latter, the emission process may be enhanced by photoemission and thermal effects.

As a second example, we estimate the efficiency of the device used by Nguyen et al. [12]. This experiment (see Figure 6) was used to determine the effective tunneling or transit time through an STM MVM junction barrier when irradiated with IR radiation. In the experiment a rectified DC current of about $1 \mathrm{nA}$ was measured as a function of tip-collector spacing (see Figure 7) for $\lambda_{\text {laser }}=1.06 \mu \mathrm{m}$. Assuming a laser power of $1-2 \mathrm{~W}$ with a beam diameter of $330 \mu \mathrm{m}$ incident on the junction, an estimated efficiency of $8-16 \%$ was obtained.

\section{Acknowledgments}

The authors thank Dr. Moon S. Chung (Ulsan University, Ulsan, S. Korea) and Dr. A. A. Lucas (Universitaires NotreDame de la Paix, Namur, Belgium) for their useful review and critique. They also wish to acknowledge useful conversations with Dr. Gary Weisel and Dr. Darin Zimmerman (Pennsylvania State University, Altoona Campus, Altoona, PA, USA). Finally, we acknowledge the assistance of and useful discussions with Dr. William Mansfield, Director of Operations of the Nanofabrication Laboratory at the Pennsylvania State University, and his associates at the facility.

\section{References}

[1] J. Alda, J. M. Rico-García, J. M. López-Alonso, and G. Boreman, "Optical antennas for nano-photonic applications," Nanotechnology, vol. 16, no. 5, pp. S230-S234, 2005.

[2] P. Bharadwaj, B. Deutsch, and L. Novotny, "Optical Antennas," Advances in Optics and Photonics, vol. 1, p. 438, 2009.

[3] N. Engheta, A. Salandrino, and A. Alù, "Circuit elements at optical frequencies: nanoinductors, nanocapacitors, and nanoresistors," Physical Review Letters, vol. 95, no. 9, Article ID 095504, 2005. 
[4] C. Rutherglen, D. Jain, and P. Burke, "Nanotube electronics for radio frequency applications," Nature Nanotechnology, vol. 4, no. 12, pp. 811-819, 2009.

[5] W. C. Brown, "The history of power transmission by radio waves," IEEE Transactions on Microwave Theory and Techniques, vol. 32, no. 9, pp. 1230-1242, 1984.

[6] B. Berland, "Photovoltaic technologies beyond the horizon: optical rectenna solar cell," Final Report, ITN Energy Systems Final Project Report, 2002.

[7] M. Bttiker and R. Landauer, "Traversal time for tunneling," Physical Review Letters, vol. 49, no. 23, pp. 1739-1742, 1982.

[8] C. B. Duke, Tunneling in Solids, Academic Press, New York, NY, USA, 1969.

[9] P. H. Cutler, T. E. Feuchtwang, T. T. Tsong, Y. Kuk, H. Nguyen, and A. A. Lucas, "Proposed use of a scanning-tunnelingmicroscope tunnel junction for the measurement of a tunneling time," Physical Review B, vol. 35, no. 14, pp. 7774-7775, 1987.

[10] L. V. Keldysh, "Ionization in thefield of a strong electromagnetic wave," Journal of Experimental and Theoretical Physics, vol. 47, pp. 1945-1957, 1964.

[11] L. V. Keldysh, "IIonization in the field of a strong electromagnetic wave," Soviet Physics, Journal of Experimental and Theoretical Physics, vol. 20, pp. 1307-1314, 1965.

[12] H. Q. Nguyen, P. H. Cutler, T. E. Feuchtwang et al., "Mechanisms of current rectification in an STM tunnel junction and the measurement of an operational tunneling time," IEEE Transactions on Electron Devices, vol. 36, no. 11, pp. 26712678, 1989.

[13] R. Gupta and B. G. Willis, "Nanometer spaced electrodes using selective area atomic layer deposition," Applied Physics Letters, vol. 90, no. 25, Article ID 253102, 2007.

[14] T. E. Sullivan, Y. Kuk, and P. H. Cutler, "Proposed planar scanning tunneling microscope diode: application as an infrared and optical detector," IEEE Transactions on Electron Devices, vol. 36, no. 11, pp. 2659-2664, 1989.

[15] K. M. Evenson, "Frequency measurements from the microwave to the visible, the speed of light, and the redefinition of the meter," in Quantum Metrology and Fundamental Physical Constants, P. H. Cutler and A. A. Lucas, Eds., Plenum, New York, NY, USA, 1983.

[16] T. E. Sullivan, Thermal and field emission effects of laser radiation on metal whisker diodes: application to infrared detection devices, Ph.D. thesis, UMI (University Microfilms International), now called Bell and Howell Information and Learning, 1977.

[17] A. Mayer, M. S. Chung, B. L. Weiss, N. M. Miskovsky, and P. H. Cutler, "Three-dimensional analysis of the geometrical rectifying properties of asymmetric metal-vacuum-metal junctions and extension for energy conversion," Physical Review B, vol. 77, no. 8, Article ID 085411, 2008.

[18] A. Mayer, M. S. Chung, B. L. Weiss, N. M. Miskovsky, and P. H. Cutler, "Three-dimensional analysis of the rectifying properties of geometrically asymmetric metal-vacuum-metal junctions treated as an oscillating barrier," Physical Review B Condensed Matter and Materials Physics, vol. 78, no. 20, Article ID 205404, 2008.

[19] A. Mayer and P. H. Cutler, "Rectification properties of geometrically asymmetric metal-vacuum-metal junctions: a comparison of tungsten and silver tips to determine the effects of polarization resonances," Journal of Physics Condensed Matter, vol. 21, no. 39, Article ID 395304, 2009.

[20] A. Mayer, M. S. Chung, B. L. Weiss, N. M. Miskovsky, and P. H. Cutler, "Simulations of infrared and optical rectification by geometrically asymmetric metal-vacuum-metal junctions for applications in energy conversion devices," Nanotechnology, vol. 21, no. 14, Article ID 145204, 2010.

[21] A. Mayer, M. S. Chung, B. L. Weiss, N. M. Miskovsky, and P. H. Cutler, "Simulations of infrared and optical rectification by geometrically asymmetric metal-vacuum-metal junctions for applications in energy conversion devices," Nanotechnology, vol. 21, no. 14, Article ID 145204, 2010.

[22] D. K. Kotter, S. D. Novack, W. D. Slafer, and P. Pinhero, "Solar nantenna electromagnetic collectors," in Proceedings of the 2 nd International Conference on Energy Sustainability (ES '08), pp. 409-415, August 2008.

[23] Y. Kuk, R. S. Becker, P. J. Silverman, and G. P. Kochanski, "Optical interactions in the junction of a scanning tunneling microscope," Physical Review Letters, vol. 65, no. 4, pp. 456459, 1990.

[24] http://physicsworld.com/cws/article/news/43556.

[25] A. A. Lucas, A. Moussiaux, M. Schmeits, and P. H. Cutler, "Geometrical asymmetry effects on tunnelling properties of point contact junctions," Communications on Physics, vol. 2, no. 6, pp. 169-174, 1977.

[26] P. J. Burke, S. Li, and Z. Yu, "Quantitative theory of nanowire and nanotube antenna performance," IEEE Transactions on Nanotechnology, vol. 5, no. 4, Article ID 1652847, pp. 314-334, 2006.

[27] L. Novotny, "Effective wavelength scaling for optical antennas," Physical Review Letters, vol. 98, no. 26, Article ID 266802, 2007.

[28] L. Novotny, "From near-field optics to optical antennas," Physics Today, vol. 64, no. 7, pp. 47-52, 2011.

[29] K. Kempa, J. Rybczynski, Z. Huang et al., "Carbon nanotubes as optical antennae," Advanced Materials, vol. 19, no. 3, pp. 421-426, 2007.

[30] A. V. Sokolov, Optical Properties of Metals, American Elsevier, New York, NY, USA, 1967.

[31] P. W. Gilberd, "The anomalous skin effect and the optical properties of metals," Journal of Physics F, vol. 12, pp. 18451860, 1982.

[32] A. K. Sarychev, V. P. Drachev, H. K. Yuan, V. A. Podolskiy, and V. M. Shalaev, "Optical properties of metal nanowires," in Nanotubes and Nanowires, pp. 92-98, usa, August 2003.

[33] A. K. Sarychev, D. A. Genov, A. Wei, and V. M. Shalaev, "Periodical arrays of optical nano-antennas," in Complex Mediums IV: Beyond Linear Isotropic Dielectrics, vol. 5218 of Proceedings of SPIE, San Diego, Calif, USA, 2003.

[34] K. R. Carver and J. W. Mink, "MICROSTRIP ANTENNA TECHNOLOGY," IEEE Transactions on Antennas and Propagation, vol. AP-29, no. 1, pp. 2-24, 1981.

[35] G. Boreman, "Infrared microantennas," in 10th Meeting on Optical Engineering in Israel, I. Shladov and S. R. Rotman, Eds., vol. 3110 of Proceedings of SPIE, p. 882, Jerusalem, Israel, 1996.

[36] I. Codreanu and G. D. Boreman, "Infrared microstrip dipole antennas - FDTD predictions versus experiment," Microwave and Optical Technology Letters, vol. 29, no. 6, pp. 381-383, 2001.

[37] C. T. Middlebrook, P. M. Krenz, B. A. Lail, and G. D. Boreman, "Infrared phased-array antenna," Microwave and Optical Technology Letters, vol. 50, no. 3, pp. 719-723, 2008.

[38] R. D. Grober, R. J. Schoelkopf, and D. E. Prober, "Optical antenna: towards a unity efficiency near-field optical probe," Applied Physics Letters, vol. 70, no. 11, pp. 1354-1356, 1997.

[39] G.S. Kino, A. Sundaramurthy, P. J. Schuck, D. P. Fromm, and W. E. Moerner, "Optical field enhancement with plasmon resonant Bowtie Nanoantennas," in Surface Plasmon 
Nanophotonics, M. Brongersma and P. Kik, Eds., chapter 9, Kluwer Acadmic Publisher, Dordrecht, The Netherlands, 2007.

[40] T. Kosako, Y. Kadoya, and H. F. Hofmann, "Directional control of light by a nano-optical Yagi-Uda antenna," Nature Photonics, vol. 4, no. 5, pp. 312-315, 2010.

[41] A. G. Curto, G. Volpe, T. H. Taminiau, M. P. Kreuzer, R. Quidant, and N. F. Van Hulst, "Unidirectional emission of a quantum dot coupled to a nanoantenna," Science, vol. 329, no. 5994, pp. 930-933, 2010.

[42] N. M. Miskovsky, S. J. Shepherd, P. H. Cutler, T. E. Sullivan, and A. A. Lucas, "The importance of geometry, field, and temperature in tunneling and rectification behavior of point contact junctions of identical metals," Applied Physics Letters, vol. 35, no. 7, pp. 560-562, 1979.

[43] N. M. Miskovsky, P. H. Cutler, T. E. Feuchtwang, S. J. Shepherd, A. A. Lucas, and T. E. Sullivan, "Effect of geometry and multiple-image interactions on tunneling and I-V characteristics of metal-vacuum-metal point-contact junctions," Applied Physics Letters, vol. 37, no. 2, pp. 189-192, 1980.

[44] S. Grafström, "Photoassisted scanning tunneling microscopy," Journal of Applied Physics, vol. 91, no. 4, p. 1717, 2002.

[45] R. M. Feenstra, J. A. Stroscio, and A. P. Fein, "Tunneling spectroscopy of the $\mathrm{Si}(111) 2 \times 1$ surface," Surface Science, vol. 181, no. 1-2, pp. 295-306, 1987.

[46] M. Dagenais, K. Choi, F. Yesikoy, A. N. Chryssis, and M. C. Peckerar, "Solar spectrum rectification using nano-antennas and tunneling diodes," in 12th Optoelectronic Integrated Circuits, L. A. El Dada and E.-H. Lee, Eds., vol. 7605 of Proceedings of SPIE, p. 76050E, San Francisco, Cali, USA, 2010.

[47] D. R. Ward, F. HÜser, F. Pauly, J. C. Cuevas, and D. Natelson, "Optical rectification and field enhancement in a plasmonic nanogap," Nature Nanotechnology, vol. 5, no. 10, pp. 732-736, 2010.

[48] X. W. Tu, J. H. Lee, and W. Ho, "Atomic-scale rectification at microwave frequency," Journal of Chemical Physics, vol. 124, no. 2, Article ID 021105, pp. 1-4, 2006.

[49] A. V. Bragas, S. M. Landi, and O. E. Martínez, "Laser field enhancement at the scanning tunneling microscope junction measured by optical rectification," Applied Physics Letters, vol. 72, no. 17, pp. 2075-2077, 1998.

[50] Y. Kuk, R.S. Becker, P. J. Silverman, and G. P. Kochanski, "Photovoltage on silicon surfaces measured by scanning tunneling microscopy," Journal of Vacuum Science \& Technology B, vol. 9, p. 545, 1991.

[51] "In comparison, an estimate of the power density for a laser with a power output of $20 \mathrm{~mW}$ and a beam diameter of about $300^{-} \mathrm{m}$ is on the order of $20 \mathrm{~W} / \mathrm{cm}^{2}$ ".

[52] M. A. Green and M. J. Keevers, "Optical properties of intrinsic silicon at $300 \mathrm{~K}$," Progress in photovoltaics, vol. 3, no. 3, pp. 189-192, 1995.

[53] B. L. Weiss, Penn State University, Altoona Campus, Altoona, PA 16601, private communication.

[54] B. Lee, E. F. Barasch, T. Mazumdar, P. M. McIntyre, Y. Pang, and H. J. Trost, "Development of knife-edge field emission cathodes on (110) silicon wafers," Applied Surface Science, vol. 67, no. 1-, pp. 66-72, 1993.

[55] A. A. Lucas, H. Morawitz, G. R. Henry et al., "Scatteringtheoretic approach to elastic one-electron tunneling through localized barriers: application to scanning tunneling microscopy," Physical Review B, vol. 37, no. 18, pp. 10708-10720, 1988.
[56] J. Z. Zhang and C. Noguez, "Plasmonic optical properties and applications of metal nanostructures," Plasmonics, vol. 3, no. 4, pp. 127-150, 2008.

[57] B. Willis, University of Connecticut, Storrs, CT 06269, private communication. 

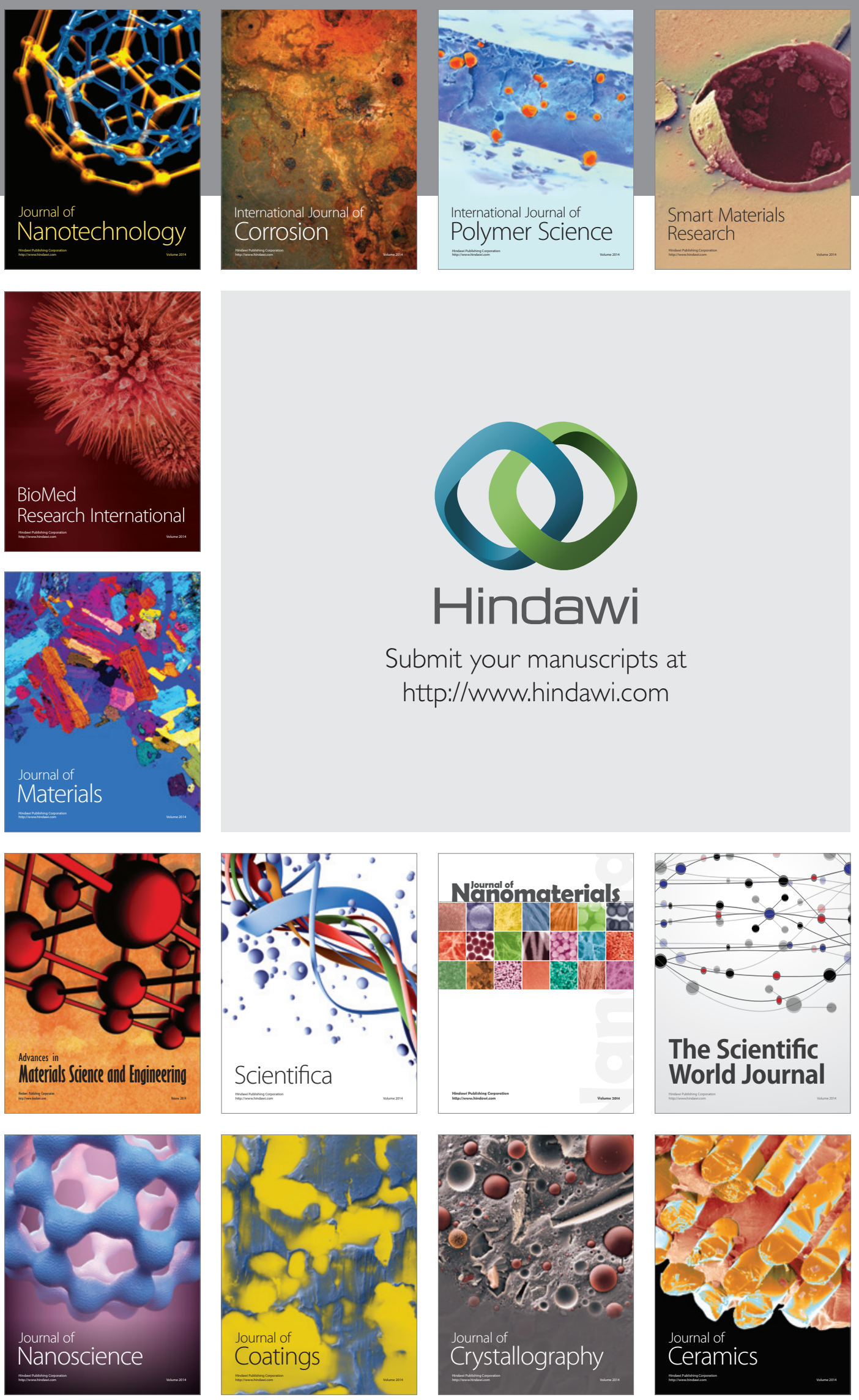

The Scientific World Journal

Submit your manuscripts at

http://www.hindawi.com

\section{World Journal}

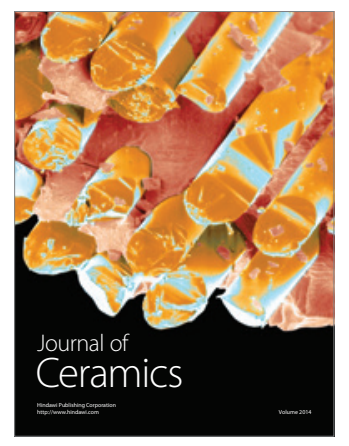

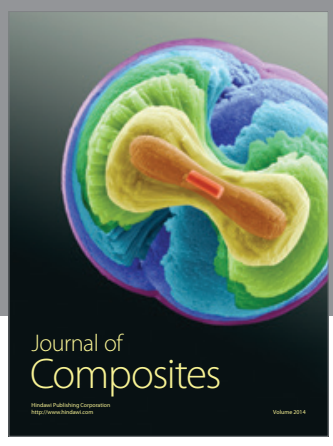
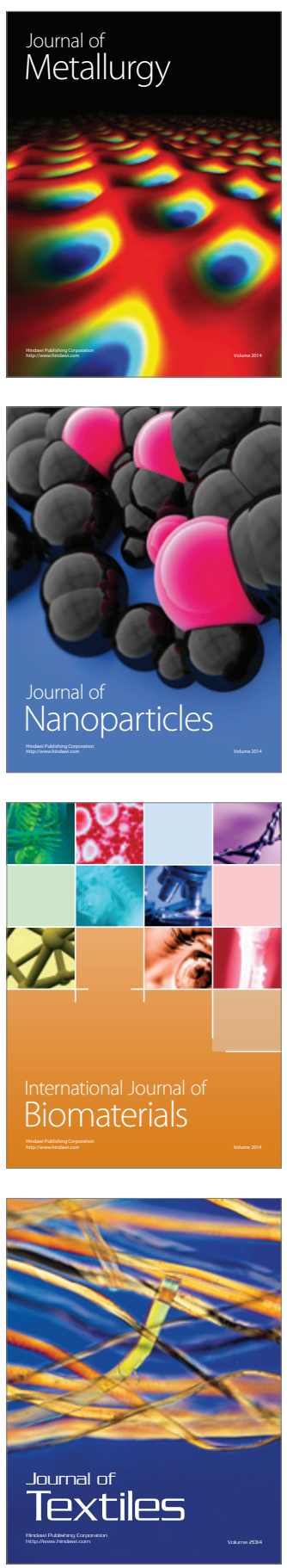